\title{
On the Theory of Electron-Transfer Reactions. VI. Unified Treatment for Homogeneous and Electrode Reactions*
}

\author{
R. A. Marcus $\dagger$ \\ Department of Chemistry, Brookhaven National Laboratory, Upton, New York, and \\ Noyes Chemical Laboratory, University of Illinois, Urbana, Illinois
}

(Received 8 March 1965)

\begin{abstract}
A unified theory of homogeneous and electrochemical electron-transfer rates is developed using statistical mechanics. The treatment is a generalization of earlier papers of this series and is concerned with seeking a fairly broad basis for the quantitative correlations among chemical and electrochemical rate constants predicted in these earlier papers. The atomic motions inside the inner coordination shell of each reactant are treated as vibrations. The motions outside are treated by the "particle description," which emphasizes the functional dependence of potential energy and free energy on molecular properties and which avoids, thereby, some unnecessary assumptions about the molecular interactions.
\end{abstract}

\section{INTRODUCTION}

$\mathbf{A}^{\mathrm{T}}$ THEORETICAL calculation of the rates of homogeneous electron-transfer reactions was described in Part I of this series ${ }^{1}$ and the method was subsequently extended to electrochemical electrontransfer rates. ${ }^{2}$ The calculation was made for reactions involving no rupture or formation of chemical bonds in the elementary electron-transfer step. In this sense these electron transfers are quite different from other types of reactions in the literature. This property, together with the assumed weak electronic interaction of the reactants, introduced several unusual features: "nonequilibrium dielectric polarization" of the solvent medium, ${ }^{3}$ possible nonadiabaticity, unusual reaction coordinate, and an approximate calculation of the reaction rate without use of arbitrary adjustable parameters.

Applications of the theoretical equations were made in several subsequent papers. ${ }^{2,4}$ The mechanism of electron transfer was later examined in more detail in Part IV using potential-energy surfaces and statistical mechanics. ${ }^{5}$ (In Part I the solvent medium outside the inner coordination shell of each reactant had been treated as a dielectric continuum. The free energy of reorganization of the medium, accompanying the formation of an activated complex having nonequilibrium

* This research was performed in part under the auspices of the U. S. Atomic Energy Commission while the author was a visiting Senior Scientist at Brookhaven National Laboratory. It was also supported by a fellowship from the Alfred P. Sloan Foundation and by a grant from the National Science Foundation. A portion of the work was performed while the author was a member of the faculty of the Polytechnic Institute of Brooklyn, and was presented in part at the 146th Meeting of the American Chemical Society held in Denver in January 1964.

† Present address: Noyes Chemical Laboratory.

1 R. A. Marcus, J. Chem. Phys. 24, 966 (1956).

2 R. A. Marcus, ONR Tech. Rept. No. 12, Project NR 051-331 (1957); cf Can. J. Chem. 37, 155 (1959) and Trans. Symp. Electrode Processes, Phila., Pa., 1959, 239-245 (1961).

${ }^{3}$ R. A. Marcus, J. Chem. Phys. 24, 979 (1956).

${ }^{4}$ R. A. Marcus, J. Chem. Phys. 26, 867, 872 (1957); Trans. N. Y. Acad. Sci. 19, 423 (1957).

B R. A. Marcus, Discussions Faraday Soc. 29, 21 (1960). dielectric polarization, was computed by a continuum method.) In Part IV, changes in bond lengths in the inner coordination shell of each reactant were also included, and the statistical-mechanical term for the free energy change in the medium outside was replaced only in the final step by its dielectric continuum equivalent.

A number of predicted quantitative correlations among the data were made on the basis of Part IV. They have received some measure of experimental support, described in Part $\mathrm{V}$ and in a recent review article. ${ }^{6,7} \mathrm{~A}$ more general basis for these correlations is described in the present paper, which also presents a unified treatment of chemical and electrochemical transfers.

The form of the final equations for the rate constants is comparatively simple, a circumstance which leads almost at once to the above correlations. (It permits extensive cancellation in computed ratios of rate constants.) This simplicity has resulted from several factors: (1) Some of the more complex aspects of the rate problem are rephrased so that they affect only a pre-exponential factor $(\rho)$ appearing in the rate constant, a factor that appears to be close to unity. (2) Little error is found to be introduced when the force constants of reactants and products are replaced by symmetrical reduced force constants. (3) An important term $(\lambda)$ in the free energy of activation is essentially an additive function of the properties of the two redox systems in the reaction.

The electron transfer rate constants can vary by many orders of magnitude: For example, known homogeneous electron-exchange rate constants vary by factors of more than $10^{15}$ from system to system, and electrochemical rate constants derived from electrochemical exchange currents vary by about $10^{8}$ at any given temperature. ${ }^{6}$ (An electron-exchange reaction is one between ions differing in their valence

\footnotetext{
${ }^{6}$ R. A. Marcus, J. Phys. Chem. 67, 853, 2889 (1963).

7 R. A. Marcus, Ann. Rev. Phys. Chem. 15, 155 (1964).
} 
state but otherwise similar.) Thus, small factors of 2 or 3 are of relatively minor importance in any theory which is intended to cover this wide range of values. Some approximations in this paper are made with this viewpoint in mind.

In the present paper classical statistical mechanics is employed for those coordinates which vary appreciably during the course of the reaction. This classical approximation is a reasonable one for orientational and translational coordinates at the usual reaction temperatures and, in virtue of the above remark, for the usual low-frequency vibrations in inner coordination shells. Because of cancellations which occur in computations of ratios of rate constants this approximation could be weakened for deriving the predicted correlations, even when the quantum corrections would not be small.

In calculations of absolute values of the electrontransfer rate constants a classical approximation will introduce some error when the necessary changes in bond lengths to effect electron transfer are so small as to be comparable with zero-point fluctuations. However, in this latter case, the vibrational contribution to the free energy of activation is itself small and does not account for any large differences in reaction rates in redox reactions which have been investigated experimentally. Hence, for our present purpose and, in the interests of simplicity, this particular possible quantum effect may be ignored.

\section{ORGANIZATION OF THE PAPER}

The paper is organized in the following way:

Individual and over-all rate constants are distinguished in Sec. 3, potential-energy surfaces for weakoverlap electron transfers are discussed in Sec. 4, and formal expressions for the rate constants are given in Sec. 5. The latter expressions arise from a generalization of activated complex theory. ${ }^{8}$ The approximate relation of certain surface integrals appearing in Sec. 5 to more readily evaluated volume integrals is described in Sec. 6, where certain complicating features are rephrased so as to cast some of the difficulties into an evaluation of one of the pre-exponential factors $\rho$. In Sec. 6 a linear dependence of an effective potential energy function (governing the configurational distributions in the activated complex) on the potential energies of reactants and products is established [Eq. (13)]. The rate constants are expressed in Sec. 7 in terms of the contribution of the coordinates of the solvent molecules in the medium and of the vibrations in the inner coordination shell of each reactant to the free energy of formation of the activated complex.

To deduce from Eq. (13) a simple dependence of the free energy of activation on differences in molecular

${ }^{8}$ R. A. Marcus, J. Chem. Phys. 41, 2624 (1964). The $U$ in the present Eqs. (1) to (3) was denoted there by $U^{\ddagger}$. parameters, the contributions of the above two sets of coordinates are treated differently (Sec. 8), since one set already has a desired property while the other does not. Changes in bond force constants accompanying electron transfer are responsible for this difference in behavior. However, it is shown later in Appendix IV that the introduction of certain "reduced force constants" circumvents the difficulty, with negligible error in typical cases. The contributions of the two sets of coordinates are computed in Secs. 9 and 10. The medium outside the inner coordination shell of each reactant is treated by a "particle description." "9,10 The latter is a considerable generalization over the customary permanent-dipole-induced-dipole treatment of polar media and serves to emphasize the functional dependence of the free energy of activation on various properties and to facilitate thereby the analysis leading to the predicted correlations.

The standard free energy of reaction and the cell potentials are introduced in Secs. 11 and 12, and are used in Sec. 13 to evaluate a quantity $(m)$ closely related to the electrochemical and chemical transfer coefficients. The final rate equations are summarized in Sec. 14.

The additive property of $\lambda$, mentioned in the previous section, is discussed in Sec. 15 and further established in Sec. 16. The significance of the characteristic scalar quantity $(m)$ appearing in the potential-energy function of the activated complex is deduced in Sec. 17. Deductions from the final equations are made in Sec.18.

In Sec. 19 the present paper is compared with earlier papers of this series, and the specific generalizations made here are described. Detailed proofs are given in various appendices. In Appendix VIII it is established that under certain conditions the correlations derived above should apply not only for rate constants of elementary steps but also for the over-all rate constant of a reaction occurring via number of complexes of the reactants with other ions in the electrolyte.

\section{INDIVIDUAL AND OVER-ALL RATE CONSTANTS}

Many chemical and electrochemical redox reagents are ions which possess inner coordination shells and which may form complexes with ions of opposite sign. Any such complex is "inner" or "outer" according as the latter ions do or do not enter the inner coordination or shell of the reactant. To a greater or lesser extent, all such complexes normally contribute to the measured rate of the redox process. For this reason both a rate

${ }^{9}$ R. A. Marcus, J. Chem. Phys. 38, 1335 (1963).

${ }_{10}$ R. A. Marcus, J. Chem. Phys. 39, 1734 (1963). The notation differs somewhat from the present paper: $\tau, U, U^{f}$, and $\rho_{s}^{\circ}$ there become $V_{o}^{\prime}, U_{o}, U_{i}$, and $\rho_{a}{ }^{\circ}$ here. A typographical error occurs in Eq. (13): The $\frac{1}{2}$ 's should be deleted. No equations deduced from (13) need correction. 
constant for the over-all reaction, involving all complexes, and a rate constant for each individual step, involving a specific complex with a given inner coordination shell or involving a specific pair of complexes in a bimolecular step, have been defined in the literature. They equal the over-all reaction rate divided by the stoichiometric concentration (or product of such concentrations in the bimolecular case), in the case of an over-all rate constant, and the reaction rate divided by the concentration of the particular complex (or product of such concentrations in the bimolecular case), in the case of an individual rate constant. Often the individual rate constants are measured experimentally. Frequently, however, only the over-all rate constant is determined in the experiment.

The derivation up to and including Sec. 6 applies to over-all as well as to individual rate constants. The Secs. 7 to 17 apply only to the individual rate constants. To calculate the over-all rate constant from the expression derived for the individual one in these latter sections, one must take cognizance of any reactions leading to the formation and destruction of the complexes and must average over the behavior of all complexes, as in Appendix VIII.

\section{POTENTIAL-ENERGY SURFACES}

The potential energy of the system is a function of the translational, rotational, and vibrational coordinates of the reacting species and of the molecules in the surrounding medium. A profile of the potentialenergy surface is given in Fig. 1 in the case of homogeneous reactions. (The related electrochemical plot is considered later.) The abscissa, a line drawn in the above many-dimensional coordinate space, represents any concerted motion of the above types leading from any spatial configuration (of all atoms) that is suited to the electronic structure of the reactants to one suited to that of the products. Surface $R$ denotes the potential-energy profile when the reacting species have the electronic structure of the reactants, and Surface $P$ corresponds to their having the electronic structure of the products. If the distance between the reacting species is sufficiently small there is the usual splitting of the two surfaces in the vicinity of this intersection of $R$ and $P$. If the electronic interaction causing the splitting is sufficient, the system will always remain on the lowest surface as it moves from left to right in Fig. 1. Thus, the system has moved from surface $R$ to surface $P$ adiabatically, in the usual sense that the corresponding motion of the atoms in the system is treated by a quantum-mechanical adiabatic method. On the other hand, if the electronic interaction causing the splitting is very weak, a system initially on Curve $R$ will tend to stay on $R$ as it passes to the right across the intersection. The probability that as a result of

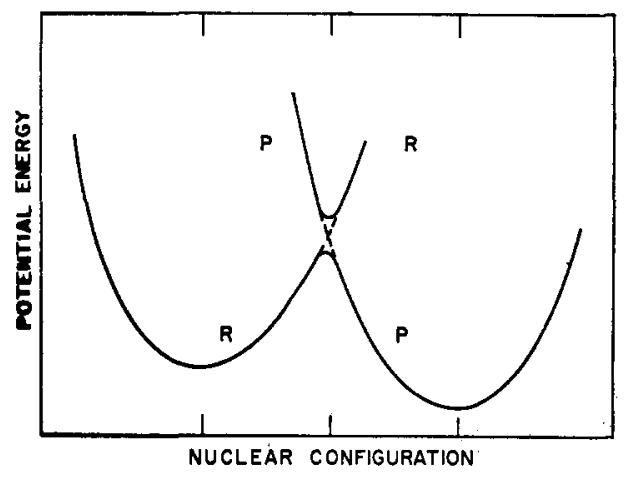

FIG. 1. Profile of potential-energy surface of reactants $(R)$ and that of products (P) plotted versus configuration of all the atoms in the system. The dotted lines refer to a system having zero electronic interaction of the reacting species. The adiabatic surface is indicated by a solid line.

this nuclear motion the system ends up on Curve $P$ is then calculated by treating this motion nonadiabatically. ${ }^{11}$

It should be noted that the system can undergo this electron transfer either by surmounting the barrier if it has enough energy or by tunneling of the atoms of the system through it if it has not. We confine our attention to the case where the systems surmount the barrier. Some atom tunneling calculations have been made, however. ${ }^{12}$

Since the abscissa in Fig. 1 is some combination of translational, rotational, and vibrational coordinates, this "reaction coordinate" is rather complex: The surfaces $R$ and $P$ intersect, and the set of configurations describing this intersection form a hypersurface in configuration space. The exact motion normal to this hypersurface depends on the part being crossed. In some parts it involves changes in bond distances in the inner coordination shells of the reactants, in other parts it involves a change of separation distance of the

11 See, for example, L. Landau, Physik. Z. Sowjetunion 1, 88 (1932); 2, 46 (1932); C. Zener, Proc. Roy. Soc. (London) A137, 696 (1932); A140, 660 (1933); C. A. Coulson and K. Zalewski, ibid. A268, 437 (1962). The present situation has been summarized in Ref. 7, where the definition of nonadiabaticity was also discussed. Reference should also have been made there to the work of. E. C. G. Stueckelberg, Helv. Phys. Acta. 5, 369 (1932); cf., H. S. W. Massey, in Encyclopedia of Physics, edited by S. Flügge (Springer-Verlag, Berlin, 1956), Vol. 36, p. 297.

${ }_{12}$ N. Sutin and M. Wolfsberg, quoted by N. Sutin, Ann. Rev. Nucl. Sci. 12, 285 (1962). These authors discussed the possibility of tunneling of the atoms in the inner coordination shell. Possible quantum effects which include atom tunneling in the medium outside this shell have been treated by V. G. Levich, and R. R. Dogonadze, Proc. Acad. Sci. USSR, Phys. Chem. Sec. [English transl. 133, 591 (1960)]; Collection Czechoslov. Chem. Comm. 26, 193 (1961) [transl., O. Boshko. University of Ottawa, Ontario.] Any conclusions concerning the contribution of atom tunneling depend in a sensitive way on the assumed values for the bond force constants and lengths in the inner coordination shell, properties on which data are now becoming available, and on the assumed value for a mean polarization frequency for the medium. [Atom tunneling is different from electron tunneling, the latter being a measure of the splitting in Fig. 1 (Ref. 7).] 


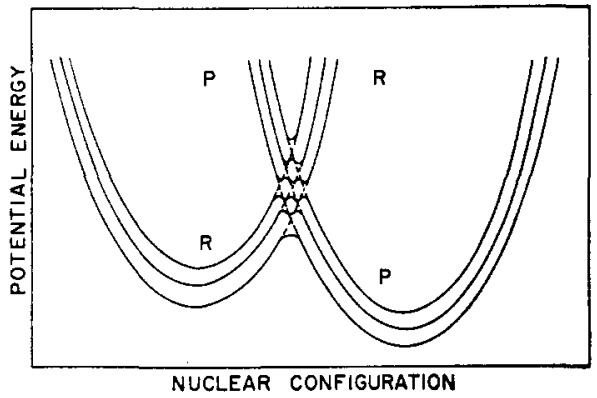

Fig. 2. Same plot as Fig. 1 but for an electrode reaction. The finite spacing between the many-electron levels of a finite electrode is enormously magnified, and only three of them are indicated. The splitting differs from level to level.

reactants, and in still others it involves reorientation of polar molecules in the medium.

Analogous remarks apply to electrode reactions except that the intersection region is more complex because of the presence of many electronic energy levels in the metal. A blown-up portion of this region is indicated in Fig. 2. The diagram consists of many potential energy surfaces, each for a many-electron state of the entire macrosystem. All the surfaces are parallel since they differ only in the distribution of electrons among "single-electron quantum states" in the metal. (Only one distribution of the electrons among these singleelectron quantum states correspond to each surface in Fig. 2 if the energy level of the entire macrosystem is nondegenerate. It corresponds to several distributions in the case of degeneracy.) There is a probability distribution of finding the macrosystem in any manyelectron energy level indicated in Fig. 2. As a consequence of a Fermi-Dirac distribution of the electrons in the metal, most electrons which are transferred to or from the many-electron energy levels in the metal will behave as though they go into or from a level which is within $k T$ of some mean energy level, and hence practically equal to it. Thus, except for the calculation of the transition probability associated with the transition from Surface $R$ to Surface $P$ in the intersection region, the situation is in effect very similar to that in Fig. 1. We return to this point in the following section. In the present paper we confine our attention in electrode reactions, as in homogeneous reactions, to reaction paths involving a surmounting of the barrier.

\section{EXPRESSION FOR THE RATE CONSTANT}

We consider any particular pair of reactants (or a reactant, in the case of intramolecular electron transfer). These "labeled" reactants may be any two given molecules in solution or one molecule and the electrode, and each may form complexes to various extents with other ions and molecules. In effect, we need to calculate the probability that the vibrational-rotational-transla- tional coordinates of the entire system are such that the system is in the vicinity of the many-dimensional intersection hypersurface in configuration space.

It is assumed below that the distribution of systems in the vicinity of the intersection region of Figs. 1 or 2 is an equilibrium one. The usual equilibrium-type derivation of the rate of a homogeneous or heterogeneous reaction in the literature employs a special form for the kinetic energy, a form consistent with the set of configurations of the activated complex being describable by a hyperplane in configuration space. A more general curvilinear formulation has been given recently. ${ }^{8}$ Upon integrating over a number of coordinates which leave the potential energy invariant one obtains (1), (2), and (3) for homogeneous bimolecular reactions, homogeneous unimolecular reactions, and heterogeneous reactions, respectively $y^{8,13}$ :

$$
\begin{aligned}
& k_{\mathrm{bi}}=(8 \pi k T)^{\frac{1}{2}} \int_{S} \frac{\exp (-U / k T) R^{2}\left(m^{\ddagger}\right)^{-\frac{1}{2}} d S}{Q}, \\
& k_{\mathrm{uni}}=\left(\frac{k T}{2 \pi}\right)^{\frac{1}{2}} \int_{S} \frac{\exp (-U / k T)\left(m^{\ddagger}\right)^{-\frac{1}{2}} d S}{Q}, \\
& k_{\mathrm{het}}=\left(\frac{k T}{2 \pi}\right)^{\frac{1}{2}} \int_{S} \frac{\exp (-U / k T)\left(m^{\ddagger}\right)^{-\frac{1}{2}} d S}{Q} .
\end{aligned}
$$

In these equations $m^{\ddagger}$ is the effective mass for motion normal to the hypersurface $S, R$ is the distance between the two reactants (normally between their centers of mass), $Q$ is the configuration integral for the reactants, and $d S$ is the area element in a many-dimensional internal coordinate space. ${ }^{13}$ Both $m^{\ddagger}$ and $R$ may vary over $S$. In (1) to (3) integration has already been performed over several coordinates, as follows: (i) in $Q$, the center of mass of each reactant; (ii) in the numerator of (1), the center of mass of one reactant and the orientation of the line of centers of the two reactants; (iii) in the numerator of (2), the center of mass of the reactant, and (iv) in the numerator of (3), the two coordinates of this center parallel to the solution-solid interface. Thus, these coordinates are to be held fixed in the internal coordinate space in (1) to (3).

In adapting these equations to electron-transfer reactions one should consider the possibility of the reaction occurring nonadiabatically and, in the case of electrodes, should consider the existence of many levels which may accept or donate an electron to a reactant in solution. In the framework of a classical treatment of the motion of the nuclei in (1) to (3), a factor $k$

\footnotetext{
${ }^{13}$ In these equations $S$ is an abbreviation for $S_{\text {int }}$ (made for brevity of notation), since several integrations over "external coordinates" have been performed and there remains only the integration over a hypersurface in internal coordinate space. ${ }^{8}$ Similarly, the symbols $S^{\prime}, V$, and $V^{\prime}$ discussed later should bear a subscript int, which is omitted here for brevity.
} 
can be shown to appear in the integrand (Appendix I); $\kappa$ is a momentum-weighted average of the transition probability from the $R$ to the $P$ surface per passage through the intersection region. (It is momentum weighted since the transition probability depends on the momentum.) $\kappa$ can vary over $S$. Normally, we take $\kappa$ as approximately equal to unity when the reactants are near each other, introducing thereby the assumption that the reaction is adiabatic.

In the case of (3) the situation is somewhat more complex because of the presence of the many electrode levels. At present there is, in the literature, no theoretical calculation of the transfer probability from a level $R$ to a continuum (essentially) of levels $P$, per passage through the intersection range, for the entire range of transfer probabilities from 0 to 1 . Such a calculation would take into account the fact that in an unsuccessful passage through the intersection region the system can also revert to other $R$ levels different from the original one. At present only the limiting case of very small transfer probability has been considered in the literature. ${ }^{14}$ In this case transfers to and from each of the levels have been treated independently using perturbation theory; they do not interfere at this limit.

When the transfer probability in electrode reactions is fairly large when ion and electrode are close, a different approach must be employed..$^{15}$ Here, we take advantage of the fact that for a metal electrode most of the electron transfers occur to and from levels near the Fermi level ${ }^{15}$ : In the terminology of a one-electron model, most of the levels several $k T$ below the Fermi level are fully occupied and cannot accept more electrons. The Boltzmann factor discourages transfer to the rather unoccupied levels several $k T$ above the Fermi level. Conversely, transfers from the occupied levels below the Fermi level are discouraged by a higher over-all energy barrier to reaction while transfer from a higher level is discouraged by the fact that most of the higher levels are unoccupied. To illustrate this point more precisely, let $n(\epsilon)$ be the density of the "oneelectron model levels" for the electrode and $f(\epsilon)$ the Fermi-Dirac distribution,

$$
f(\epsilon)=\left\{\exp \left[\left(\epsilon-\vec{\mu}_{e}\right) / k T\right]+1\right\}^{-1},
$$

where $\epsilon$ is the energy of one of these levels and where $\bar{\mu}_{e}$ is the electrochemical potential of electrons in the metal. Both $\epsilon$ and $\bar{\mu}_{e}$ depend on the electrostatic potential of the metal $\phi$ :

$$
\epsilon=\epsilon(0)-e \phi ; \quad \bar{\mu}=\mu-e \phi,
$$

\footnotetext{
${ }^{14}$ R. R. Dogonadze and Y. A. Chizmadzhev, Proc. Acad. Sci. USSR, Phys. Chem. Sec., English Transl. 144, 463 (1962), 145, 563 (1962); V. G. Levich and R. R. Dogonadze, Intern. Comm. Electrochem. Thermodyn. Kinet., 14th Meeting, Moscow (1963), preprints. This work is reviewed in Ref. 7.

${ }_{16}^{16}$ This approximation was used but not discussed in Ref. 2.
}

where $\epsilon(0)$ is the value of $\epsilon$ at $\phi=0$ and $\mu$ is the chemical potential. ${ }^{16}$

The probability that electron transfer from the electrode to the ion or molecule in solution will occur from a "one-electron model" level of energy $\epsilon$ would be expected to depend on $\epsilon$ by a factor roughly proportional to

$$
n(\epsilon) f(\epsilon) \exp (\epsilon / 2 k T),
$$

the third factor arising in the region where the "electrochemical transfer coefficient" is 0.5 , a common value. ${ }^{6}$ Since $n(\boldsymbol{\epsilon})$ is a weak function of $\epsilon$ the last two factors in (6) largely determine the most probable value of $\epsilon$. The maximum of (6) is then easily shown to occur at $\epsilon=\bar{\mu}_{e}$. Similarly, contribution to electron transfer from an ion in solution to a particular level $\epsilon$ would be expected to vary with $\epsilon$ as in

$$
n(\epsilon)[1-f(\epsilon)] \exp (-\epsilon / 2 k T),
$$

which also has a maximum at $\epsilon=\bar{\mu}_{e}$, of course.

Because of this circumstance (that most contributions arise from levels $\epsilon$ near $\bar{\mu}_{e}$ ), we approximate the situation in Fig. 2 by replacing the set of Surfaces $R$ by one surface and $P$ by another surface, corresponding to an electronic energy in the electrode given by $\bar{\mu}_{e}$ as above. ${ }^{15}$ If electron transfer accompanies each passage through the intersection region in Fig. 2 the reaction is referred to as "adiabatic," purely by analogy with the term in the homogeneous reaction. The reaction rate is given by (3), where the equation of $S$ depends on the electrostatic potential. On the other hand, when the transfer probability per passage is very weak a term $\kappa$ should be introduced in the integral, $\kappa$ being a velocity-weighted transition probability appropriately summed over all energy levels in the electrode (Appendix I). A value for $\kappa$ in this weak interaction limit has been discussed elsewhere. ${ }^{7}$ When a complete calculation for the transfer probability from and to a continuum of electrode levels becomes available it can be used to estimate $\kappa$. Normally, however, we assume the electrode reaction to be "adiabatic" and so take $\kappa \cong 1$ on the average.

\section{RELATION OF THE SURFACE INTEGRALS} (1) TO (3) TO VOLUME INTEGRALS

Although some deductions can be made from the surface integrals in (1) to (3) when the equation of the intersection surface $S$ is simple, we find it convenient to express the surface integral in terms of volume one. The same aim was followed in Part IV but in a less precise way. The principal equation derived in this section is (26), which is later used in conjunction with Eqs. (1) to (3) to obtain an expression for $k_{\text {rate. }}$

${ }^{16}$ For example, C. Herring, and M. H. Nichols, Rev. Mod. Phys. 21, 185 (1949). 
Let $U^{r}$ be the potential-energy function for the reactant and $U^{p}$ be that for the products. As mentioned earlier the intersection of the $R$ and $P$ surfaces in Fig. 1 (and 2) forms a hypersurface in configuration space. This hypersurface is called the "reaction hypersurface." Its equation is given by (8). It is a hypersurface in the entire coordinate space and also in the internal coordinate space since (8) is independent of the external coordinates ${ }^{8}$

$U^{r}-U^{p}=0 \quad$ (for points on reaction hypersurface).

This surface is a member of a family of hypersurfaces in configuration space, represented by (9), where $c$ is a constant:

$$
U^{r}-U^{p}=c .
$$

The surface (8) can be obtained from the surface (9) by lowering the $P$ surface in Figs. 1 or 2 by an amount $c$.

We employ a coordinate system $q^{1}$ to $q^{n}$ used in the derivation of (1) to (3) and recall that one coordinate, $q^{N}$, in the internal coordinate space was chosen to be a coordinate constant on the hypersurface (8). Let $q^{N}$ be zero there. In fact, each member of the family of hypersurfaces (9) is made a coordinate hypersurface for $q^{N}$.

We consider any of the integrals in (1) to (3), include the factor $\kappa$ in the integrand, and write $d S$ as $d S^{\prime} d R{ }^{17}$ The factor $\kappa$ depends primarily on $R$. In the following expression the same symbol $\kappa$ is used to denote this $\kappa$, averaged over $S^{\prime}{ }^{18}$ Each of the integrals in (1) to (3) can be rewritten as

$$
\int_{R} \kappa R^{\alpha}\left[\int_{S^{\prime}} \exp \left(-\frac{U}{k T}\right)\left(m^{\ddagger}\right)^{-\frac{1}{2}} d S^{\prime}\right] d R,
$$

where $\alpha$ is 2, 0 , or 0 according to whether (1), (2), or (3) is the equation involved.

We wish to relate the above integral over $S^{\prime}$ to a volume integral (11) over the internal coordinate space at fixed $R$, as in (18) and finally as in $(26)^{19}$ :

$$
\int_{V^{\prime}} \exp \left(-\frac{U^{*}}{k T}\right) d V^{\prime}
$$

where $U^{*}$ is a function to be determined; $R^{\alpha} d V^{\prime}$ is an element of volume of this internal coordinate space at fixed $R^{8}$

${ }^{17}$ This factoring of $d S$ (or as it was called there $d S_{\text {int }}$ ) was described in Ref. 8.

${ }^{18}$ The $\kappa$ appearing in (10) is now a symbol representing

$$
\int \kappa\left(m^{\ddagger}\right)^{-1} \exp \left(\frac{-U}{k T}\right) d S^{\prime} / \int\left(m^{\ddagger}\right)^{-\frac{1}{2}} \exp \left(\frac{-U}{k T}\right) d S^{\prime},
$$

where $\kappa$ is the original kappa.

${ }^{19}$ These "internal coordinates" were defined ${ }^{8}$ as those coordinates for which integration was not performed in obtaining (1) to (3).
To establish (18), we first note from Appendix II that the distribution in volume which is centered on $S^{\prime}$ (but not confined to $S^{\prime}$, of course) is $f^{*}$, given by $(12)^{20}$

$$
f^{*}=\exp \left(-\frac{U^{*}}{k T}\right) / \int \exp \left(-\frac{U^{*}}{k T}\right) d V^{\prime}
$$

where

$$
U^{*}=U^{r}+m\left(U^{r}-U^{p}\right)
$$

and $m$ is a parameter which varies with the coordinate $R$ and which is determined in Sec. 13. On $S^{\prime}$, one sees from (8), $U^{*}$ equals $U^{r}$ for any given $R$.

We then recall from Ref. 8 that $d V^{\prime}$ and $d S^{\prime}$ are related by (14), and we introduce a quantity $I\left(q^{N}, R\right)$ defined by (15):

$$
\begin{gathered}
d V^{\prime}=\left(a^{N N}\right)^{-\frac{1}{2}} d S^{\prime} d q^{N}, \\
\exp \left[-I\left(q^{N}, R\right)\right]=\int \exp \left(-\frac{U^{*}}{k T}\right)\left(a^{N N}\right)^{-\frac{1}{d}} d S^{\prime},
\end{gathered}
$$

where $a^{N N}$ is conjugate to an element $a_{N N}$ in the line element of the many-dimensional configuration space. On recalling from Ref. 8 that $m^{\ddagger}$ equals $a^{N N} / g^{N N}$, where $g^{N N}$ is conjugate to an element $g_{N N}$ in the line element of the corresponding mass-weighted configuration space, the $S^{\prime}$ integral in (10) can then be rewritten as in (16), where $\left\langle\left(g^{N N}\right)\right.$ is a suitable average over $S^{\prime 21}$

$$
\int \exp \left(-\frac{U}{k T}\right)\left(m^{\ddagger}\right)^{-\frac{1}{2}} d S^{\prime}=\left\langle\left(g^{N N}\right)^{\frac{1}{2}}\right\rangle \exp [-I(0, R)] .
$$

In deriving (16) we have also used the fact that $U^{*}$ equals $U^{r}$ on $S^{\prime}$.

Finally, the integral in (11) can be rewritten as $\int \exp \left[-I\left(q^{N}, R\right)\right] d q^{N}$, in virtue of (14) and (15). On the basis of a Gaussian expansion Eq. (17) can be derived (post).

$$
\begin{aligned}
\int \exp \left[-I\left(q^{N}, R\right)\right] d q^{N} & \\
= & {\left[2 \pi I^{\prime \prime}(0, R)\right]^{\frac{1}{2}} \exp [-I(0, R)], }
\end{aligned}
$$

where $I^{\prime \prime}(0, R)$ is $d^{2} I\left(q^{N}, R\right) / d q^{N 2}$, evaluated on $S^{\prime}$ (and hence at $q^{N}=0$ ). One then obtains, from (10),

\footnotetext{
${ }^{20}$ If, for any $R$, a distribution function $f^{*}$ is stated to be centered on $S^{\prime}$, we mean that it is centered on the set of configurations which lie at the intersection of the hypersurface $S$ and of the hypersurface $R=$ constant. Occasionally, in some part of the internal coordinate space the two hypersurfaces may be "cotangential," but this circumstance does not alter the argument. At these parts of space the value of $U^{r}$ equals $U^{p}$ and (12) becomes "exact" for computing relative probabilities of various configurations, rather than approximate.

${ }_{21}\left\langle\left(g^{N N}\right)^{\frac{1}{3}}\right\rangle$ in $(16)$ is defined as

$\int\left(g^{N N}\right)^{\prime}\left(a^{N N}\right)^{-t} \exp \left(\frac{-U}{k T}\right) d S^{\prime} / \int\left(a^{N N}\right)^{-t} \exp \left(\frac{-U}{k T}\right) d S^{\prime}$
} 
(16), and (17),

$$
\begin{aligned}
\int_{R} \kappa R^{\alpha}\left[\int_{S^{\prime}} \exp \left(-\frac{U}{k T}\right)\left(m^{\ddagger}\right)^{-\frac{1}{a}} d S^{\prime}\right] d R \\
\quad=\int_{R} \frac{\kappa R^{\alpha}\left\langle\left(g^{N N}\right)^{\frac{1}{2}}\right\rangle \exp \left[-F^{*}(R) / k T\right]}{\left[2 \pi I^{\prime \prime}(0, R)\right]^{\frac{1}{2}}} d R,
\end{aligned}
$$

where $F^{*}(R)$ is the configurational free energy of a system having the potential-energy function $U^{*}$ for this separation distance $R$

$$
\exp \left(-\frac{F^{*}(R)}{k T}\right)=\int \exp \left(-\frac{U^{*}}{k T}\right) d V^{\prime}
$$

To complete the proof of (18) we must verify (17). We recall from the definition of $q^{N}$ that $U^{r}-U^{p}$ depends only on $q^{N}$ on any hypersurface (9). To ensure centering of the system on $S^{\prime}$, i.e., at $q^{N}=0, m(R)$ is to be selected so that (20) is satisfied:

$$
\left\langle U^{r}\right\rangle=\left\langle U^{p}\right\rangle,
$$

where \langle\rangle denotes average with respect to the distribution function $f^{*}$. On using (12), (14), and (15), Eq. (20) becomes

$$
\int \exp \left[-I\left(q^{N}, R\right)\right]\left(U^{r}-U^{p}\right) d q^{N}=0 .
$$

Because of the centering of $f^{*}$, expansion of $I\left(q^{N}, R\right)$ about $q^{N}=0$ is permissible, as is one of $U^{r}-U^{p}$

$$
\begin{aligned}
I\left(q^{N}, R\right)=I(0, R) & +q^{N} I^{\prime}(0, R) \\
& +\left[\left(q^{N}\right)^{2} / 2 !\right] I^{\prime \prime}(0, R)+\cdots, \\
U^{r}-U^{p}= & 0+q^{N}\left(U^{r}-U^{p}\right)^{\prime}+\cdots,
\end{aligned}
$$

where ' indicates a derivative with respect to $q^{N}$, evaluated at $q^{N}=0$. We retain only leading terms in each case. Insertion of (22) and (23) into (21) followed by integration reveals that $I^{\prime}(0, R)$ vanishes. Introduction of (22) into the left-hand side of (17) then establishes (17).

Some of the terms in (18) can be expressed in terms of quantities of more immediate physical significance. It may be shown from (12), (14), (15), (22) and the vanishing of $I^{\prime}(0, R)$ that

$$
I^{\prime \prime}(0, R)=\left\langle\left(\delta q^{N}\right)^{2}\right\rangle^{-1},
$$

where $\left\langle\left(q^{N}\right)^{2}\right\rangle$ is the mean-square deviation of $q^{N} .{ }^{22}$ The mean-square deviation of the perpendicular distance $s$ from the reaction hypersurface is given by (25)

\footnotetext{
22 This average, $\left\langle\left(\delta q^{N}\right)^{2}\right\rangle$, is defined as $\int\left(\delta q^{N}\right)^{2} f^{*} d V^{\prime}$. It is
} readily shown that $\left\langle q^{N}\right\rangle$ vanishes. for small s's:

$$
\left\langle(\delta s)^{2}\right\rangle=\left\langle\left(a^{N N}\right)^{-1}\left(\delta q^{N}\right)^{2}\right\rangle=\left\langle\left(a^{N N}\right)^{-1}\right\rangle\left\langle\left(\delta q^{N}\right)^{2}\right\rangle,
$$

where $\left\langle\left(a^{N N}\right)^{-1}\right\rangle$ is a suitable average of $\left(a^{N N}\right)^{-1} .23$

We make use of the fact that $\left\langle\left(g^{N N}\right)^{\frac{1}{2}}\right\rangle\left\langle\left(a^{N N}\right)^{-1}\right\rangle^{\frac{1}{2}}$ has units of (mass) ${ }^{-\frac{1}{2}}$, and denote it by $\left(m^{*}\right)^{-\frac{1}{3}}$, and that the integrand in (18) has a maximum at some value of $R$, denoted in (26) by $R$. [When $R$ becomes large $\kappa$ tends to zero and when $R$ is small the van der Waals' repulsion makes $F^{*}(R)$ large.] On treating the integrand as a Gaussian function of $R,(18)$ becomes

$$
\begin{aligned}
\int_{R} \kappa R^{\alpha}\left[\int_{S^{\prime}} \exp (\right. & \left.\left.-\frac{U}{k T}\right)\left(m^{\ddagger}\right)^{-\frac{1}{2}} d S^{\prime}\right] d R \\
& =\kappa \rho R^{\alpha}\left(m^{*}\right)^{-\frac{1}{2}} \exp \left[-F^{*}(R) / k T\right],
\end{aligned}
$$

where $\kappa$ is evaluated at this value of $R$ and where $\rho$ is a ratio (27) whose value should be of the order of magnitude of unity:

$$
\rho=\left[\left\langle(\delta R)^{2}\right\rangle /\left\langle(\delta s)^{2}\right\rangle\right]^{2},
$$

where $\left\langle(\delta R)^{2}\right\rangle$ is the mean square deviation in the value of $R$; $\rho$ and $\kappa$ can be calculated from more specific models when the various integrals defining them can be evaluated.

\section{RATE CONSTANT IN TERMS OF $\Delta F^{*}$}

Let $F^{r}$ be the configurational free energy associated with the $Q$ of Eqs. (1) to (3) as in (28). Thereby, it is the free-energy contribution for an equilibrium distribution of " $V$ ' coordinates" when the reactants are very far apart but fixed in position,

$$
F^{r}=k T \ln Q .
$$

Let $F^{r}(R)$ be the corresponding quantity when the reactants are a distance $R$ apart. We then have

$$
w^{r}=F^{r}(R)-F^{r}
$$

where $w^{r}$ can be called the reversible work to bring the reactants from fixed positions infinitely far apart to the cited separation distance.

We also introduce $\Delta F^{*}(R)$ :

$$
\Delta F^{*}(R)=F^{*}(R)-F^{r}(R) .
$$

Equations (1) to (3) for $k_{\text {rate }}$ now yield (31) to (33), when (26) and (28) to (30) are used,

$$
\begin{aligned}
k_{\mathrm{bi}} & =\kappa \rho Z_{\mathrm{bi}} \exp \left(-w^{r} / k T\right) \exp \left[-\Delta F^{*}(R) / k T\right], \\
k_{\mathrm{uni}} & =\kappa \rho\left(k T / 2 \pi m^{*}\right)^{\frac{1}{2}} \exp \left(-\Delta F^{*} / k T\right), \\
k_{\text {het }} & =\kappa \rho Z_{\text {het }} \exp \left(-w^{r} / k T\right) \exp \left[-\Delta F^{*}(R) / k T\right],
\end{aligned}
$$

${ }^{23}$ This average is defined here as
$\int\left(a^{N}\right)^{-1}\left(\delta q^{N}\right)^{2} \exp \left(\frac{-U^{*}}{k T}\right) d V^{\prime} / \int\left(\delta q^{N}\right)^{2} \exp \left(\frac{-U^{*}}{k T}\right) d V^{\prime}$. For the proof that $d s^{2}$ equals $\left(a^{N N}\right)^{-1}\left(d q^{N}\right)^{2}$, see Ref. 32, Appendix III. 
where $Z_{\mathrm{bi}}$ and $Z_{\text {het }}$ are given by

$$
Z_{\mathrm{bi}}=\left(8 \pi k T / m^{*}\right)^{\frac{1}{2}} R^{2} \quad Z_{\mathrm{het}}=\left(k T / 2 \pi m^{*}\right)^{\frac{1}{2}} .
$$

[In Eq. (32) $\Delta F^{*}$ is simply $F^{*}-F^{r}$, there being only one reactant.] $Z_{\mathrm{bi}}$ is in fact the collision number of two uncharged species in solution when they have unit concentration, when their reduced mass is $m^{*}$, and when their collision diameter is $R$. $Z_{\text {het }}$ is the collision number of an uncharged species with unit area of an interface (here, the electrode), when it has unit concentration and when its mass is $m^{*}$.

$F^{*}$ and $F^{r}$ in (31) to (33) involve an integration over the orientation of each reactant. The integrand in $F^{r}$ is independent of these coordinates and, in the case of the "outer-sphere electron-transfer mechanism" discussed here, the integrand in $F^{*}$ is assumed to be independent of them also. (For purposes of deriving many of the correlations in Sec. 16, this assumption could be weakened because of cancellations.) Integration over these coordinates is regarded as having been performed in (31) to (34), since the orientational factors now cancel in $\Delta F^{*}(R)$. Thus, in the subsequent calculation of $F^{*}$ and $F^{r}$ each reactant may be regarded as fixed not only in position, as before, but in orientation also.

\section{DISTRIBUTION FUNCTION AND THE FREE ENERGY}

The main purpose of this section is the derivation of Eqs. (47) to (49).

Equation (19) for $F^{*}(R)$ can be rewritten as in (35), with the aid of $(12),(13)$, and (20):

where

$$
F^{*}(R)=\left\langle U^{r}\right\rangle+k T\left\langle\ln f^{*}\right\rangle
$$

$$
\left\langle U^{r}\right\rangle=\int U^{r} f^{*} d V^{\prime}, \quad\left\langle\ln f^{*}\right\rangle=\int\left(\ln f^{*}\right) f^{*} d V^{\prime} .
$$

Since $-k\left\langle\ln f^{*}\right\rangle$ is the configurational entropy of a system having the distribution function $f^{*}$ and since $\left\langle U^{r}\right\rangle$ is the mean potential energy of a nonequilibrium system having a potential-energy function $U^{r}$ but a distribution function of $f^{*}$ inappropriate to this $U^{r}$, we see that $F^{*}(R)$ is also equal to the configurational free energy of this nonequilibrium system.

In obtaining an expression for $F^{*}(R)$ it is convenient to divide, as one usually does in related problems, the internal coordinates at the given $R$ into two groups: $V^{\prime}{ }_{i}$ coordinates describing the positions of the atoms in the inner coordination shells of the reactants, and $V^{\prime}{ }_{o}$ coordinates describing the positions of the atoms of the medium relative to each other and to those in the inner coordination shells. It is also convenient to write $U$ as the sum of two terms, $U_{i}$ and $U_{o}$, one describing the intramolecular interactions of the atoms in each coordination shell, the other describing the interactions of the atoms of the medium with each other and with those of the inner coordination shells. Thus, $U_{i}$ depends only on the $V^{\prime}{ }_{i}$ coordinates; $U_{o}$ depends primarily on the $V^{\prime}{ }_{0}$ coordinates, but also depends on the $V^{\prime}{ }_{i}$ ones,

$$
U=U_{i}+U_{o}
$$

The quantities $U_{i}^{*}$ and $U_{0}^{*}$ are defined in terms of $U_{i}^{r}$, etc., to be given by (13), with $i$ and $o$ subscripts, respectively. Then, $U^{*}$ is the sum of $U_{i}^{*}$ and $U_{o}^{*}$.

The volume element $d V^{\prime}$ is written as

$$
d V^{\prime}=d V^{\prime} d V^{\prime}{ }_{o}
$$

where $d V^{\prime}{ }_{i}$ is defined as the product of the differentials $\left(\Lambda_{i} d q^{i}\right)$ of the $V^{\prime}{ }_{i}$ coordinates. Thereby, $d V^{\prime}{ }_{0}$ contains the Jacobian appearing in $d V^{\prime}$. It may vary, therefore, with the $V^{\prime}{ }_{i}$ coordinates.

In calculating $F^{*}$ and $F^{r}$ we may evaluate the integrals appearing in them by first integrating over the $V^{\prime}{ }_{0}$ coordinates and then over the $V^{\prime}{ }_{i}$ ones. This procedure is convenient since the $V^{\prime}{ }_{i}$ ones perform small oscillations while the others can undergo considerable fluctuations. With this procedure in mind, we define new quantities $f_{i}^{*}$ and $f_{o}{ }^{*}$, the former depending only on the $V^{\prime}{ }_{i}$ coordinates, the latter depending on the $V_{o}^{\prime}$ coordinates and parametrically on the $V^{\prime}{ }_{i}$ ones:

$$
\begin{aligned}
& f_{o}{ }^{*}=\exp \left[\left(\chi_{o}^{*}-U_{o}^{*}\right) / k T\right], \\
& f_{i}{ }^{*}=\exp \left(-\frac{\bar{U}_{i}^{*}}{k T}\right) / \int \exp \left(-\frac{\bar{U}_{i}{ }^{*}}{k T}\right) d{V^{\prime}}^{\prime},
\end{aligned}
$$

where

$$
\begin{gathered}
\bar{U}_{i}^{*}=U_{i}^{*}+\chi_{o}^{*} \\
\exp \left(-\frac{\chi_{o}^{*}}{k T}\right)=\int \exp \left(-\frac{U_{o}^{*}}{k T}\right) d V_{o}^{\prime} .
\end{gathered}
$$

One then obtains

$$
f^{*}=f_{o}^{*} f_{i}{ }^{*} .
$$

Quantities $f_{o}^{r}, f_{i}^{r}, \bar{U}_{i}^{r}$, and $\chi_{o}^{r}$ can be defined, by replacing the * by an $r$ superscript in (39) to (42). However, $\chi_{o}{ }^{r}$ is simply $F_{o}{ }^{r}$, the $V^{\prime}{ }_{o}$ contribution to the configurational free energy of the reactants for the given value of the $V^{\prime}{ }_{i}$ coordinates

$$
\exp \left(-\frac{F_{o}^{r}}{k T}\right)=\int \exp \left(-\frac{U_{o}^{r}}{k T}\right) d V^{\prime}{ }_{0}
$$

We also introduce $F_{o}{ }^{*}$, defined by

$$
F_{o}^{*}=\left\langle U_{o}^{r}\right\rangle_{*_{o}}+k T\left\langle\ln f_{o}^{*}\right\rangle_{*_{o}},
$$

where the average \langle\rangle$_{*_{0}}$ is computed with respect to $f_{0}{ }^{*}$. $F_{o}{ }^{*}$ is the $V^{\prime}{ }_{o}$ contribution to the free energy of the nonequilibrium system having the potential energy function $U^{r}$ and the distribution function $f_{0}{ }^{*}$. The first and second terms in (45) are the energy and entropy contributions, respectively.

To obtain an expression for $\bar{U}_{i}^{*}$, the function largely controlling the $V_{i}^{\prime}$ coordinate distribution, we first 
obtain (46) by introduction of (39) into (45) and by use of (13) with subscript $o$ 's added. Equations (41), (46), (37) and, with subscript $i$ 's added, (13) then yield (47), since $U_{i}{ }^{r}$ and $U_{i}{ }^{p}$ are independent of the $V^{\prime}{ }_{0}$ coordinates:

$$
\begin{aligned}
& \chi_{o}^{*}=F_{o}^{*}+m\left\langle U_{o}^{r}-U_{o}{ }^{p}\right\rangle_{*_{o}}, \\
& \bar{U}_{i}{ }^{*}=U_{i}^{r}+F_{o}{ }^{*}+m\left\langle U^{r}-U^{p}\right\rangle_{*_{o} .}
\end{aligned}
$$

Equations (35), (37), (43), and (45) yield (48a), when one notes that $U_{i}^{r}$ and $f_{i}{ }^{*}$ are independent of the value of the $V^{\prime}{ }_{0}$ coordinates. Equation $(48 \mathrm{~b})$ then follows from (20), (47), and (48a):

$$
\begin{aligned}
F^{*}(R) & =\left\langle U_{i}{ }^{r}+F_{o}^{*}\right\rangle_{*_{i}}+k T\left\langle\ln f_{i}^{*}\right\rangle_{*_{i}}, \\
& =\left\langle\bar{U}_{i}{ }^{*}+k T \ln f_{i}{ }^{*}\right\rangle_{* i},
\end{aligned}
$$

where the average \langle\rangle$_{*_{i}}$ is computed with respect to the distribution function $f_{i}^{*}$.

The free energy $F^{r}(R)$, given by

$$
-k T \ln \int \exp \left(-\frac{U^{r}}{k T}\right) d V^{\prime}
$$

evaluated at $R$, can also be shown to be given by expressions similar to (48) but with the asterisks replaced by $r$ 's

$$
F^{r}(R)=\left\langle U_{i}^{r}+F_{o}^{r}\right\rangle_{r i}+k T\left\langle\ln f_{i}^{r}\right\rangle_{r i}
$$

To evaluate $k_{\text {rate, }}$ we compute $\Delta F^{*}$ from (30), (48), and (49), and use (47).

The similarity of (48) and (49) and later of (51) and (52) is an example of the fact that properties of the $[r]$ system can be obtained from those of the $[*]$ system by setting $m=0$. The origin of this behavior is seen in the original Eq. (13) defining the $[*]$ system.

\section{VIBRATIONAL CONTRIBUTION TO $\Delta F^{*}(R)$}

While it is not necessary to introduce the harmonic approximation, the expressions are appreciably simplified by it. There is evidence that the approximation is adequate for many reactions of interest.

It is recalled that the generalized coordinates were denoted by $q^{j}$. Let the first $n_{i}$ of these be vibrational coordinates of the reacting species, i.e., the $V_{i}{ }^{\prime}$ coordinates, and let $q *^{j}$ denote the value of the $j$ th vibrational $q^{j}$ occurring at the minimum of $\bar{U}_{i}^{*}$. We have

$$
\begin{aligned}
\bar{U}_{i}^{*} & =\bar{U}_{i}^{*}(\mathbf{Q} *)+\frac{1}{2} \sum_{j, k=1}^{n i} f_{j k}^{*}\left(q^{j}-q *^{j}\right)\left(q^{k}-q *^{k}\right) \\
& =\bar{U}_{i}^{*}(\mathbf{Q} *)+\frac{1}{2}\left(\mathbf{Q}^{T}-\mathbf{Q} *^{T}\right) \cdot \mathbf{F}^{*}(\mathbf{Q}-\mathbf{Q} *),
\end{aligned}
$$

where $\mathbf{Q}-\mathbf{Q}$ * denotes a column vector whose elements are $q^{j}-q *^{j}$. F* denotes a square matrix whose elements are $f_{i j}{ }^{*}$. The superscript $T$ denotes a transpose (a row vector here), and the dot indicates the scalar product of this row vector with the column vector $F^{*}\left(\mathbf{Q}-\mathbf{Q}_{*}\right)$.
On multiplying numerator and denominator of (40) by $\exp \left[U_{i}^{*}\left(\mathbf{Q}_{*}\right) / k T\right]$, introducing this expression for $f_{i}{ }^{*}$ into $(48 \mathrm{~b})$, then using (50), integrating, ${ }^{24}$ and finally introducing an expression for $\bar{U}_{i}\left(\mathbf{Q}^{*}\right)[\mathbf{E q} .(47)$ evaluated at $\mathbf{Q}=\mathbf{Q}^{*}$ ], Eq. (51) follows:

$$
\begin{array}{r}
F^{*}(R)=U_{i}{ }^{r}(\mathbf{Q} *)+F_{o}{ }^{*}(\mathbf{Q} *)-m\left\langle U^{r}(\mathbf{Q} *)-U^{p}(\mathbf{Q} *)\right\rangle_{*_{o}} \\
-\frac{1}{2} k T \ln \left[(2 \pi k T)^{n_{i}} /\left|f_{j k}{ }^{*}\right|\right], \quad(51)
\end{array}
$$

where $\left|f_{j k}{ }^{*}\right|$ is the determinant of the $f_{j k}{ }^{*}$ s.

If $q_{r}{ }^{j}$ denotes the value of a vibrational $q^{j}$ occurring at the minimum of $U_{i}^{r}$, it can be shown that $F^{r}$ is given by (52) after a quadratic expansion of $\bar{U}_{i}^{r}(\mathbf{Q})$ about $\mathbf{Q}_{\tau}$,

$F^{r}(R)=U_{i}{ }^{r}\left(\mathbf{Q}_{r}\right)+F_{o}{ }^{r}\left(\mathbf{Q}_{r}\right)-\frac{1}{2} k T \ln \left[(2 \pi k T)^{n_{i}} /\left|f_{j k}{ }^{r}\right|\right]$,

where

$$
f_{j k}{ }^{r}=\left(\partial^{2} \bar{U}_{i}^{r} / \partial q^{j} \partial q^{k}\right) \text { at } \mathbf{Q}=\mathbf{Q}_{r} .
$$

Equation (54) is then obtained from (51) and (52) by noting that $\left\langle U^{r}\left(\mathbf{Q}_{*}\right)-U^{p}\left(\mathbf{Q}_{*}\right)\right\rangle_{*_{o}}$ vanishes (Appen$\operatorname{dix} \mathrm{V})$, that $U_{i}^{r}$ equals $U_{i}^{r}-F_{o}^{r}$ at any $\mathbf{Q}$, and that $\bar{U}_{i}^{r}\left(\mathbf{Q}^{*}\right)$ can be expanded about the value of $\bar{U}_{i}{ }^{r}$ at $\mathbf{Q}_{r}$ :

$$
\begin{aligned}
& F^{*}(R)-F^{r}(R)=\frac{1}{2}\left(\mathbf{Q}^{*}-\mathbf{Q}_{r}{ }^{T}\right) \cdot \mathbf{F}^{r}\left(\mathbf{Q}^{*}-\mathbf{Q}_{r}\right) \\
& +\Delta F_{o}{ }^{*}\left(\mathbf{Q}^{*}\right)+\frac{1}{2} k T \ln \left(\left|f_{j k}{ }^{*}\right| /\left|f_{j k}{ }^{r}\right|\right),
\end{aligned}
$$

where

$\Delta F_{\circ}^{*}(\mathbf{Q})=F_{o}^{*}(\mathbf{Q})-F_{o}^{r}(\mathbf{Q}) \quad($ at any given $R)$.

It is shown later that at any given $R$ and $\mathbf{Q} \Delta F_{o}^{*}(\mathbf{Q})$ equals $m^{2} \lambda_{o}(\mathbf{Q})$, where $\lambda_{o}(\mathbf{Q})$ is given by (69), and that $\Delta F_{o}{ }^{* p}(\mathbf{Q})$, which is $F_{o}^{* p}(\mathbf{Q})-F_{o}^{r}(\mathbf{Q})$, equals $(m+1)^{2} \lambda_{o}(\mathbf{Q})$. We then obtain (56) from $(47)^{25}$

$$
\bar{U}_{i}^{*}=\bar{U}_{i}^{r}+m\left(\bar{U}_{i}{ }^{r}-\bar{U}_{i}^{p}\right)-m(m+1) \lambda_{o}(\mathbf{Q}) .
$$

Since $\bar{U}_{i}{ }^{*}$ is a minimum at $\mathbf{Q}=\mathbf{Q} *$, the first variation in $U_{i}^{*}$ vanishes for any arbitrary infinitesimal $\delta \mathbf{Q}$. In Appendix VI it is found that $\lambda_{o}$ may be neglected in obtaining

$\delta \mathbf{Q}^{T} \cdot\left[(m+1) \mathbf{F}^{r}\left(\mathbf{Q}_{*}-\mathbf{Q}_{r}\right)-m F^{p}\left(\mathbf{Q}_{*}-\mathbf{Q}_{p}\right)\right]=0$.

Since the $\delta q^{i}$ are selected to be independent, the coefficient of $\delta \mathbf{Q}^{T}$ vanishes. Hence,

$$
\mathbf{Q} *=\left[(m+1) \mathbf{F}^{r}-m \mathbf{F}^{p}\right]^{-1}\left[(m+1) \mathbf{F}^{r} \mathbf{Q}_{r}-m \mathbf{F}^{p} \mathbf{Q}_{p}\right],
$$

and the first term in (54) becomes

$$
\frac{1}{2}\left(\mathbf{Q}^{T}-\mathbf{Q}_{r}^{T}\right) \cdot \mathbf{F}^{r}\left(\mathbf{Q} *-\mathbf{Q}_{r}\right)=\frac{1}{2} m^{2} \Delta \mathbf{Q}^{T} \cdot \mathbf{F} \Delta Q,
$$

${ }^{24}$ We use Eq. (2) in R. Bellman, Introduction to Matrix Analysis (McGraw-Hill Book Company, Inc., New York, 1960), p. 96 , to obtain the last term of $(51)$.

${ }^{25}$ On recalling the definition of $\bar{U}_{i}^{r}$ and $\bar{U}_{i}{ }^{p}$, and adding and subtracting $m k T\left\langle\ln f_{0}\right\rangle_{0}$ it follows that $\bar{U}_{i}^{*}$ in $(47)$ can be written as $(m+1) \bar{U}_{i}{ }^{r}-m \bar{U}_{i}{ }^{p}$ plus $\Delta F_{0}{ }^{*}+m\left(\Delta F_{0}{ }^{*}-\Delta F_{0}{ }^{* p}\right)$. Equation (56) then follows. 
where

$$
\begin{aligned}
\Delta \mathbf{Q} & =\mathbf{Q}_{p}-\mathbf{Q}_{r}, \\
\mathbf{F} & =\mathbf{F}^{p}\left[(m+1) \mathbf{F}^{r}-m \mathbf{F}^{p}\right]^{-1} \mathbf{F} r\left[(m+1) \mathbf{F}^{r}-m \mathbf{F}^{p}\right]^{-1} \mathbf{F}^{p},
\end{aligned}
$$

and the equality of $\mathbf{F}^{r}, \mathbf{F}^{p}$, and $\left[(m+1) \mathbf{F}^{r}-m \mathbf{F}^{p}\right]^{-1}$ with their transposes have been used.

On differentiating (56) twice and noting that an a posteriori calculation shows that the last term in (56) may be ignored in the differentiation we find (62), for use in the $\ln$ term in (51)

$$
f_{j k}^{*}=\partial^{2} \bar{U}_{i}^{*} / \partial q^{j} \partial q^{k}=(m+1) f_{j k}{ }^{r}-m f_{j k}{ }^{p} .
$$

Later it is shown that Eqs. (54) and (59) can be simplified considerably to a good approximation by introduction of symmetrical and antisymmetrical functions of the force constants and then neglecting terms involving the antisymmetrical ones

$$
\begin{aligned}
k_{j k} & =2 f_{j k}{ }^{r} f_{j k}^{p} /\left(f_{j k}{ }^{r}+f_{j k}^{p}\right), \\
l_{j k} & =\left(f_{j k}{ }^{r}-f_{j k^{p}}\right) /\left(f_{j k}{ }^{r}+f_{j k}{ }^{p}\right) .
\end{aligned}
$$

The first of these quantities was chosen so as to have dimensions of a force constant and the second of these so as to be dimensionless.

\section{ORIENTATION AND OTHER CONTRIBUTIONS TO $\Delta F^{*}$}

For purposes of generality we employ the particle description of the potential energy in a macrosystem..$^{9,10}$ It introduces fewer assumptions than those normally used in condensed polar media. Because of its comparative generality it also permits a simultaneous formulation of the theory of homogeneous intermolecular electron transfers, electron transfers at electrodes, and intramolecular electron transfers. In this description the system consists of particles each of which is a reacting molecule or any electrode present, the latter including as part of it any strongly bound layer of ions or solvent. The remainder of the system, the medium, can then be regarded as one giant particle.

The potential energy is the sum of an intraparticle term (the energy when the particles are isolated, each having the given intraparticle coordinates) and an interparticle term (the energy change when the particles are brought together for the given values of the intraparticle coordinates). The solvent particle possesses a "cavity" for each reactant particle, which the latter fills when they are brought together.

The intraparticle terms below contain the electronic and potential energy of the reactants and of the medium. The interparticle term is, in the first approximation, the sum of interparticle polar terms and of interparticle electron correlation (i.e., exchange repulsion and London dispersion) energies. ${ }^{9}$ It can then be expanded in powers of the permanent charge density $\rho_{a}{ }^{\circ}$ of the reactants. The usual approximations in the literature correspond to neglect of powers higher than the second, together with the assumption of specific forms for these terms. ${ }^{9}$

In terms of the symbols $U_{i}$ and $U_{o}$ introduced earlier, we have

where

$$
U=U_{i}+U_{o}
$$

$$
U_{o}=U(0)+U(1)+U(2) .
$$

In (37) $U_{i}$ is the intraparticle term for the reactants and $U_{o}$ is the sum of the intraparticle term for the medium and of the interparticle term. $U(0), U(1)$, and $U(2)$ depend functionally on zeroth, first, and second powers of $\rho_{a}{ }^{\circ}$ and, respectively, on second, first, and zeroth powers of $\rho_{M}{ }^{\circ}$, the permanent charge density of the medium..$^{9} U(0)$ also contains the intraparticle term for the medium and the electron correlation interparticle term. $U_{i}$ and $\rho_{a}{ }^{\circ}$ depend only on the intraparticle coordinates, $V^{\prime}{ }_{i}$, of the reactants, and $\rho_{M}{ }^{\circ}$ depends only on those of the medium, $V^{\prime}{ }^{9}{ }^{9}$

The distribution function $f_{0}{ }^{*}$ defined in (39) can be shown to be similar to that which occurs when the permanent charge distribution on a reactant $A$ is $\rho_{a}{ }^{\circ}$, given by (66) for all $A$ :

$$
\rho_{a}{ }^{\circ *}=\rho_{a r}{ }^{\circ}+m\left(\rho_{a r}{ }^{\circ}-\rho_{a p}{ }^{\circ}\right),
$$

where $\rho_{a r}{ }^{\circ}$ is the permanent charge distribution of Molecule A when it is actually a reactant and $\rho_{a p}{ }^{\circ}$ is that when it is a product. The proof is given in Appendix III and utilizes the facts that $U(1)$ is a linear functional of $\rho_{a}{ }^{\circ}$ and that $U(2)$ is insensitive to the usual translational-rotational fluctuations in condensed media, for reasons noted there, unlike the $U(0)$ and $U(1)$. Normally, as will be seen later, $m$ will be close to $-\frac{1}{2}$.

The $V_{0}^{\prime}$ contribution to the free energy of formation of a system with a nonequilibrium $V^{\prime}{ }_{0}$ distribution, $\Delta F_{o}{ }^{*}$, at any given $R$ and at any given $\mathbf{Q}$, has been evaluated elsewhere on the basis of the particle description described above and of an assumption of (at most) partial electric saturation ${ }^{10}$ :

$$
\Delta F_{o}^{*}=F_{m(r-p)}^{\mathrm{op}}-F_{m(r-p)} .
$$

In (67) $F^{\mathrm{op}}$ and $F$ denote the polar contributions to the free energies of two hypothetical equilibrium and dielectrically unsaturated systems, each having a $\rho_{a}{ }^{\circ}$ equal to $m\left(\rho_{a r}{ }^{\circ}-\rho_{a p}{ }^{\circ}\right)$ on each reactant. The first system is an "optical polarization" system," i.e., a system whose medium responds to these $\rho_{a}{ }^{\circ}$ 's only via an electronic polarization. The second system responds via all polarization terms. Both $F^{\mathrm{op}}$ and $F$ are quadratic functions of the $m\left(\rho_{a r}{ }^{\circ}-\rho_{a p}{ }^{\circ}\right)$ 's.

It can be shown ${ }^{26}$ that $F^{\mathrm{op}}-F$ depends on the square

${ }^{26}$ According to Eqs. (10) and (11) of Ref. $10 F^{\circ D}-F$ equals $\left[\left\langle U(1)^{2}\right\rangle-\langle U(1)\rangle^{2}\right] / 2 k T$. The latter depends only on the second power of the charge distribution, since $U(1)$ is a linear functional of the first power. 
of the permanent charge distribution on the reactants, in this case $m\left(\rho_{a r}{ }^{\circ}-\rho_{a p}{ }^{\circ}\right)$. We may then describe the dependence of $\Delta F_{0}{ }^{*}$ by

$$
\Delta F_{o}^{*}=m^{2} \lambda_{o}
$$

where

$\lambda_{o}=\left\langle\left[U_{o}^{r}(1)-U_{o}^{p}(1)-\left\langle U_{o}^{r}(1)-U_{o}^{p}(1)\right\rangle\right]^{2}\right\rangle / k T$

and the averaging function is ${ }^{27}$ :

$\exp \left(-\frac{\left(U_{o}^{r}+U_{o}^{p}\right)}{2 k T}\right) d V_{\circ}^{\prime} / \int \exp \left(-\frac{\left(U_{o}^{r}+U_{o}^{p}\right)}{2 k T}\right) d V_{o}^{\prime}$

To use Eq. (68) and those derived earlier, an expression is needed for $m$. It is derived below after some preliminary analysis involving the standard free energy of reaction, the electrochemical cell potential, and the activation overpotential.

\section{STANDARD FREE ENERGY OF REACTION}

The configurational free energy of the system when the reacting species are labeled reactant molecules, fixed in position but far apart, was denoted by $F^{r}$. The corresponding quantity when the pair refers to labeled product molecules was denoted by $F^{p}$. The momentum and translational contributions of each member of the reacting pair to the free energy of the initial state cancels that in the final state in these reactions involving no change in total number of moles of redox species. Thus, the difference $F^{p}-F^{r}$ is equal to the free energy of reaction when a pair of labeled reactants form a pair of labeled products in the prevailing medium.

This free energy of reaction in the prevailing medium can be expressed in terms of "standard" chemical potentials. The chemical potential $\mu_{i}$ can be written as $\mu_{i}{ }^{\circ \prime}+k T \ln c_{i}$, where $\mu_{i}{ }^{\circ \prime}$ is the "standard" chemical potential, defined here as the value of $\mu_{i}$ at $c_{i}=1$. Because of the labeling, $F^{p}-F^{r}$ does not contain a contribution from entropy of mixing of the reactants. Since it is these mixing terms which contribute the $k T \ln c_{i}$ to $\mu_{i}$, we therefore have

$$
F^{p}-F^{r}=\sum_{p} \mu_{p}{ }^{\circ \prime}-\sum_{r} \mu_{r}{ }^{\circ \prime},
$$

where $\sum_{p}$ and $\sum_{r}$ denote summation over products and reactants. There are one or two terms in each sum, according as the reaction is unimolecular or bimolecular.

The right-hand side of (71) is $\Delta F^{\circ}$, the "standard" free energy of reaction for the prevailing medium,

${ }^{27}$ If the dielectric unsaturation approximation is used, one can show ${ }^{10}$ that $\left(U_{o^{r}}+U_{o}^{p}\right) / 2$ would be replaced by $U(0)$ in Eq. (70). Within the range of validity of the partial dielectric saturation approximation, the average of the fluctuation term (69) would be the same if $\left(U_{o}^{r}+U_{0}^{p}\right) / 2$ were replaced by $U_{0}^{*}$, by $U_{o}^{r}$ or by $U_{o}{ }^{p}$. We have simply selected some mean value for the exponent, symmetrical in $r$ and $p$. temperature, and pressure. Hence,

$$
F^{p}-F^{r}=\Delta F^{\circ} \text {. }
$$

It equals $-k T \ln K$, where $K$ is the equilibrium "constant" measured under these conditions. Both $\Delta F^{\text {of }}$ and $K$ can vary with electrolyte concentration, with temperature, and with pressure.

\section{ACTIVATION OVERPOTENTIAL AND ELECTRODE-SOLUTION POTENTIAL DIFFERENCE}

For electrode systems, the counterpart of (72) is obtained by considering the free energy of Reaction (73) for a labeled molecule at any fixed position in the body of the solution, but far from the electrode, $M$

$$
\operatorname{Red}+M=\mathrm{Ox}+M(n e),
$$

where $\mathrm{Ox}$ and Red denote the oxidized and reduced forms of the labeled molecule in the body of the solution. This free energy change, which accompanies the transfer of $n$ electrons from the ion or molecule to the electrode at a mean energy level discussed in Sec. 5, has a number of contributions, such as one from the change in electronic energy, one from the change in ion-solvent interactions in the vicinity of the ion, and one from the change in vibrational energy. Let $F^{r}$ now denote the configurational free energy of the system containing the electrode and a labeled reactant, the latter fixed in a position far outside the electrode double layer. Let $F^{p}$ denote the corresponding quantity when labeled molecule is a product, the electrode having gained $n$ electrodes as in (73).

The term $F^{p}-F^{r}$ is linear in the metal-solution potential difference, as may be seen from the discussion in Sec. 5, and thereby in the half-cell potential $E$. ( $E$ is defined to be the half-cell potential corrected for any ohmic drop and concentration polarization.) We have then

$$
F^{p}-F^{r}=\Delta+n e E,
$$

where $\Delta$ is independent of $E$, and where we have used a standard convention regarding the sign of $E$. [This convention is one which makes Reaction (73) increasingly spontaneous with increasing positive $E_{o}{ }^{\prime}$, a quantity defined later.]

Because of the labeling the entropy-of-mixing term of the oxidized molecules and that of the reduced molecules are again absent in $F^{r}$ and $F^{p}$. When the system is at electrochemical equilibrium and when the probability of finding the labeled species as a reactant is the same as that for finding it as a product, $F^{p}-F^{r}$ must vanish. Also, $E$ then has its equilibrium value, which is $E_{o}^{\prime}$ for the case of equal concentrations of the labeled species. [ $E_{o}$ ' is the "standard" oxidation potential or, as it is sometimes called, the formal oxidation potential of the half-cell; $E_{o}^{\prime}$ is defined in terms of the 
equilibrium half-cell potential $E_{e}$ by (75) for any ratio of concentrations (Red)/(Ox)]

$$
E_{e}=E_{o}^{\prime}+(k T / n e) \ln [(\mathrm{Red}) /(\mathrm{Ox})] .
$$

One then obtains, from (74),

Hence,

$$
0=\Delta+n e E_{o}{ }^{\prime} .
$$

$$
F^{p}-F^{r}=n e\left(E-E_{o}{ }^{\prime}\right) .
$$

We observe from (77) that $E-E_{o}{ }^{\prime}$, rather than the activation overpotential $E-E_{e}$, plays the role of the "driving force" in these reactions. The same role is played by $\Delta F^{\circ \prime}$ in the homogeneous reaction.

In terms of formal electrochemical potentials of the product and reactant ions and in terms of the electrochemical potential of the electrons in the electrode we have, incidentally, for Reaction (73),

$$
F^{p}-F^{r}=\bar{\mu}_{p}{ }^{\circ \prime}-\bar{\mu}_{r}{ }^{\circ \prime}+n \bar{\mu}_{e} .
$$

\section{EQUATION FOR $m$}

We first note that $\Delta F^{\circ}$ can be written as the algebraic sum of the following terms: The free energy change when the reactants are brought together to the separation distance $R$, $w^{r}$; the free energy of reorganization of the reacting system at this $R, \Delta F^{*}$; the free energy difference of reactants and products in this reorganized state, which equals

$$
\left(\left\langle U^{p}+k T \ln f^{*}\right\rangle-\left\langle U^{r}+k T \ln f^{*}\right\rangle\right)
$$

because of cancellation of momentum and of translational contributions; minus the free energy of reorganization of the product system at this $R$ to the above state, $-\Delta F^{* p}$; and minus the free energy change when the products are brought together to the separation distance $R,-w^{p}$. Thus, (79) is obtained when (20) is used,

\section{(Homogeneous)}

$$
\Delta F^{\circ \prime}=w^{r}+\Delta F^{*}(R)-\Delta F^{* p}(R)-w^{p} .
$$

The electrochemical equation corresponding to (79) is (80), as one may show from (77),

(Electrochemical)

$$
n e\left(E-E_{o}^{\prime}\right)=w^{r}+\Delta F^{*}(R)-w^{p}-\Delta F^{* p}(R) .
$$

Here, $\Delta F^{* p}$ is obtained from $\Delta F^{*}$, and $w^{p}$ from $w^{r}$ by interchanging $r$ and $p$ superscripts and, at the same time, interchanging $-m$ and $m+1$. To establish this result it suffices to note from (13) that $U^{*}$ and all its associated properties are unaffected by such a transformation, but the properties of the reactants become those of the products.

Upon introducing Eqs. (54) and (60) for $\Delta F^{*}(R)$, using (68) for $\Delta F_{o}^{*}\left(Q_{*}\right)$, and upon introducing the counterpart of this equation for $\Delta F^{* p}(R)$, the equation for $m$ is obtained. The final equations for the reaction rate become quite simple when one notes that to an excellent approximation terms involving the $l_{j k}$ 's defined in (64) can be neglected. The proof is given in Appendix IV.

\section{SUMMARY OF FINAL EQUATIONS}

On using the results of Appendix IV and referring to Eqs. (31) to (33), it is found that the rate constant for a bimolecular homogeneous reaction or a unimolecular electrochemical reaction is given by

$$
k_{\text {rate }}=\kappa \rho Z \exp \left(-\Delta F^{*} / k T\right),
$$

where $Z$ is given by (34), $\Delta F^{*}$ by (81) and (82), and $\rho$ by $(27)$.

The rate constant of an intramolecular electron transfer reaction, on the other hand, is given by $\mathrm{Eq}$. (32), with $\Delta F^{*}$ given by (81) but with the work terms $w^{r}$ and $w^{p}$ omitted:

Homogeneous:

$$
\Delta F^{*}=\frac{w^{r}+w^{p}}{2}+\frac{\lambda}{4}+\frac{\Delta F^{\circ \prime}}{2}+\frac{\left(\Delta F^{\circ \prime}+w^{p}-w^{r}\right)^{2}}{4 \lambda},
$$

Electrochemical:

$$
\begin{aligned}
\Delta F^{*}=\frac{w^{r}+w^{p}}{2}+\frac{\lambda}{4}+ & \frac{n e\left(E-E_{o}^{\prime}\right)}{2} \\
& +\frac{\left(n e E-n e E_{o}{ }^{\prime}+w^{p}-w^{r}\right)^{2}}{4 \lambda}
\end{aligned}
$$

In (81) and (82) $\lambda$ is given by

$$
\lambda=\lambda_{i}+\lambda_{o}
$$

where $\lambda_{i}$ is given by (84) and $\lambda_{o}$ is given by (69) at $\mathbf{Q}=\mathbf{Q}$ *. On introducing the symmetrical force constants one finds $\mathbf{Q}_{*}=\mathbf{Q}_{r}+m\left(\mathbf{Q}_{r}-\mathbf{Q}_{p}\right)$. Since $\lambda_{o}$ depends but weakly on $\mathbf{Q}_{*}$ and since $m$ is usually close to $-\frac{1}{2}$, it suffices to evaluate $\lambda_{o}$ at $\mathbf{Q}_{*}=\frac{1}{2}\left(\mathbf{Q}_{\mathrm{r}}+\mathbf{Q}_{p}\right)$ in the typical case. This result is used in deriving Eq. (88a),

$$
\lambda_{i}=\frac{1}{2} \sum_{j, k} k_{j k} \Delta q_{j} \Delta q_{k} \text {. }
$$

The reduced force constants $k_{j k}$ are defined in (63) and the $\Delta q_{j}$ 's are differences in equilibrium values of bond coordinates (e.g., independent bond lengths and angles), $q_{j}^{r}-q_{j}{ }^{p}$.

It is expected that typically $\rho$ should be about unity. As noted earlier, $Z$ is essentially the collision number, being about $10^{11}$ liter $\mathrm{mole}^{-1} \cdot \mathrm{sec}^{-1}$ and $10^{4} \mathrm{~cm} \mathrm{sec}^{-1}$ for homogeneous and electrochemical reactions, respectively.

In Ref. 6 the above equations were written in an 
equivalent form

$$
\begin{gathered}
\Delta F^{*}=w^{r}+m^{2} \lambda, \\
\text { Homogeneous: }-(2 m+1) \lambda=\Delta F^{\circ}+w^{p}-w^{2},
\end{gathered}
$$

Electrochemical: $-(2 m+1) \lambda=n e\left(E-E_{o}{ }^{\prime}\right)+w^{p}-w^{r}$.

The value of $m$ defined by (86) can be shown to differ very slightly from that in the preceding sections, due to the approximation of neglect of the $l_{j k}$ 's, but the final equations obtained when (86) is introduced into (85) are identical with (81) and (82).

According to Eqs. (81) and (82) $\Delta F^{*}$ depends on $\Delta F^{\circ \prime}$ or on $n e E$ according to $(87 \mathrm{a})$ and $(87 \mathrm{~b})$ when $w^{r}$ and $w^{p}$ are held constant.

$$
\begin{aligned}
\left(\partial \Delta F^{*} / \partial \Delta F^{\circ \prime}\right)_{w} & =\frac{1}{2}(1 / 2 \lambda)\left(\Delta F^{\circ \prime}+w^{p}-w^{r}\right), \\
\left(\partial \Delta F^{*} / \partial n e E\right)_{w} & =\frac{1}{2}+(1 / 2 \lambda)\left(n e E-n e E_{o}^{\prime}+w^{p}-w^{r}\right) .
\end{aligned}
$$

We refer to these slopes as "transfer coefficients at constant work terms." The second term in (87a) and (87b) can be calculated when $\lambda$ is known, and this in turn can be estimated from the experimental value of $\Delta F^{*}$ at $\Delta F^{\circ \prime}=0$, or at $E=E_{o}^{\prime}$ using (81) or (82), when the work terms can be estimated or are negligible. 'Typically, this second term is found to be small, so that these "transfer coefficients" are then 0.5.

Equations (87a) and (87b) are based on the neglect of the antisymmetrical functions $l_{j k}$ defined in (64). When these functions are not neglected, the transfer coefficient is not exactly 0.5 for zero $\left(\Delta F^{\circ \prime}+w^{p}-w^{r}\right) / \lambda$ or zero $\left(n e E-n e E_{o}{ }^{\prime}+w^{p}-w^{r}\right) / \lambda$, but is given instead by Eq. (A14) in Appendix IV. When these two sources of deviation from a 0.5 value are small, we may add them and so obtain (87c) and (87d) instead of (87a) and $(87 \mathrm{~b})$ :

$$
\begin{aligned}
\left(\partial \Delta F^{*} / \partial \Delta F^{\circ \prime}\right)_{w}=\frac{1}{2}+(1 / 2 \lambda)\left(\Delta F^{\circ \prime}+w^{p}-w^{r}+\frac{1}{2} \lambda_{i}\right. & \left.\left\langle l_{s}\right\rangle\right), \\
\left(\partial \Delta F^{*} / \partial n e E\right)_{w}=\frac{1}{2}+(1 / 2 \lambda) & (87 \mathrm{c}) \\
\quad \times\left(n e E-n e E_{o}^{\prime}+w^{p}-w^{r}+\frac{1}{2} \lambda_{i}\left\langle l_{s}\right\rangle\right) . & (87 \mathrm{~d})
\end{aligned}
$$

As noted in Appendix IV the $\left\langle l_{s}\right\rangle$ term could cause a deviation from the 0.5 value by 0.04 when the force constants in the products are all twice as large (or all twice as small) as the corresponding ones in the reactants and when $\lambda_{i} / \lambda$ is about $\frac{1}{2}$. Smaller differences in force constants would lead to even smaller deviations than 0.04 . This source of deviations would be difficult to detect experimentally, since there are other sources of deviation as well. In the case of homogeneous reactions, force constants on one reactant may stiffen and those in the other weaken, so that the average value of $\left\langle l_{s}\right\rangle$ may be even less than that for the above case, and the deviation from the 0.5 value arising from this source correspondingly smaller.

In summary, the transfer coefficient at constant w's is expected to be close to $\frac{1}{2}$, reflecting a type of symmetry of the $R$ and $P$ surfaces in the vicinity of the reaction hypersurface (compare also Sec. 17). A source of deviation from this symmetry arises from a difference in corresponding force constants in reactants and products. It appears as an $\left\langle l_{s}\right\rangle$ term in (87) and has been shown to be small. A second source of deviation arises when the $R$ or $P$ surface is appreciably lower than the other, and is reflected in the presence of the $\Delta F^{\circ \prime}$ and $n e\left(E-E_{o}{ }^{\prime}\right)$ terms in (87). This source of deviation, too, is normally small. The leading term in (87),$\frac{1}{2}$, arises from the quadratic nature of both the $V^{\prime}{ }_{o}$ and the $V^{\prime}{ }_{i}$ contributions to $\Delta F^{*}$.

\section{PROPERTIES OF THE REORGANIZATION TERM $\lambda$}

For use in subsequent correlations, we examine an additivity property of $\lambda$ and the relation between the values of $\lambda$ in related homogeneous and electrochemical systems. We consider first the (hypothetical) situation when $R$ is very large, so large that the force field from one reactant does not influence the other. On noting that $\lambda_{o}$ is given by (69) and that the fluctuations around each reactant are now independent (large $R$ ), $\lambda_{o}$ can be written as the sum of two independent terms, one per reactant. It then follows that when $R$ is large the value of $\lambda_{o}$ for a reaction between reactants from two different redox systems $A$ and $B, \lambda_{o}{ }^{a b}$, is the arithmetic mean of the values $\lambda_{0}{ }^{a a}$ and $\lambda_{o}{ }^{b b}$ of the respective systems:

$$
\lambda_{0}^{a b}=\frac{1}{2}\left(\lambda_{0}^{a a}+\lambda_{0}^{b b}\right) \quad(R \text { large }) .
$$

Furthermore, in the electrochemical case there is only a contribution from one ion (assuming that any distortion of atomic structure of the electrode yields only a relatively minor contribution to $\Delta F_{0}{ }^{*}$ ). Denoting the values of $\lambda_{o}$ for the electrochemical redox system $A$ and for the homogeneous redox system $A$ by $\lambda_{o}{ }^{\text {el }}$ and $\lambda_{o}{ }^{\text {ex }}$ respectively, we have

$$
\lambda_{0}^{\mathrm{ex}}=2 \lambda_{0}^{\mathrm{el}} \quad(R \text { large }) .
$$

Relations similar to (88a) and (88b) also hold for $\lambda_{i}$, independent of $R$, as may be seen from (84): Part of the sum for $\lambda_{i}$ is over the bonds of the first reactant and the remainder is over those of the second one. While the $k_{j k}$ 's of one reactant in the activated complex depends slightly on the fact that there is a neighboring reactant, this influence is taken to be weak.

In the absence of specific interactions, Eqs. (88a) and (88b) would also hold for smaller $R$, since in the equation for $\lambda_{o}$ each ion would merely see another charge, $-m \Delta e$, and the surrounding medium, in both the homogeneous and electrode cases. In the homo- 
geneous case, the $-m \Delta e$ is centered on the other ion. In the electrode case it is an image charge on the electrode..$^{28}$ To obtain some estimate of deviations from (88a) due to differences in ion size (one type of "specific effects") we examine in the next section the evaluation of $\lambda_{o}$ in the dielectric continuum approximation.

\section{DIELECTRIC CONTINUM ESTIMATE AT $\Delta \boldsymbol{F}_{o}{ }^{*}$}

The present section on a continuum estimate of $\Delta F_{o}{ }^{*}$ is included partly for what it can reveal approximately about certain aspects of the statistical mechanical value for $\Delta F_{o}^{*}$ and partly for making some approximate numerical calculations. It does not form a necessary part of the present electron-transfer theory itself, of course, for the latter rests on statistical mechanics.

We note that $\Delta F_{o}{ }^{*}$ can be regarded as the sum of two contributions, $\Delta F^{*}{ }_{\mathrm{sol}}$ and $\Delta F^{*}{ }_{\mathrm{atm}} \cdot \Delta F^{*}{ }_{\mathrm{sol}}$ is defined as the contribution if the atmospheric ions have not adapted themselves to the change $m\left(\rho_{a r}^{\circ}-\rho_{a p}{ }^{\circ}\right)$, and $\Delta F^{*}{ }_{\text {atm }}$ is defined as the contribution due to their adaptation ("reorganization"). $\Delta F^{*}{ }_{\text {sol }}$ in an electrolyte medium will not have exactly the same value it has at infinite solution, since the local dielectric properties near the reactants will be altered somewhat by the presence of salt.

These two contributions are estimated in Appendix VII by treating the medium as a dielectric continuum, the ion atmosphere as a continuum, and the reactants as spheres, and by neglecting dielectric image effects. ${ }^{29}$ We obtain (89) and (90) for the value of $\Delta F^{*}{ }_{\text {sol }}$ for a medium treated as dielectrically unsaturated continuum outside the inner coordination shell of each reactant. If partial saturation occurs, Eq. (67) still applies. ${ }^{3}$ If one then introduces "differential" rather than "integral" dielectric constants, as defined in the Appendix, and treats them approximately as constants Eqs. (89) and (90) again apply but now $D_{\text {op }}$ and $D_{8}$ are mean values of these differential constants

Homogeneous:

$$
\Delta F_{\mathrm{sol}}^{*}=m^{2}(n e)^{2}\left(\frac{1}{2 a_{1}}+\frac{1}{2 a_{2}}-\frac{1}{R}\right)\left(\frac{1}{D_{\mathrm{op}}}-\frac{1}{D_{s}}\right),
$$

\footnotetext{
${ }^{28}$ Quantum-mechanical calculations in support of the classical image law are given by R. G. Sachs and D. L. Dexter, J. Appl. Phys. 21, 1304 (1950). At a distance of $5 \AA$ from the electrode the computed energy of an ion in vacuum may be estimated from their results to be about $8 \%$ higher than that estimated from the image law. Experimental evidence for the validity of the image law at distances of $5 \AA$ has been offered by L. W. Swanson and R. Gomer, J. Chem. Phys. 39, 2813 (1963) (cf. p. 2835).

${ }_{29}$ The dielectric image contribution to $\Delta F_{\text {sol }}{ }^{*}$ is estimated to be negligible: It makes essentially no contribution to the value of $F_{m(r-p)}{ }^{\text {op }}$ since this hypothetical system has a low diectric constant equal to the optical dielectric constant throughout. Its contribution to $F_{m}(r-p)$ is only about $8 \%$ of the value of the term containing $1 / D_{s}$ in (90). Since this term is only a negligible fraction of the $1 / D_{\mathrm{op}}$ term in $(90)$, the dielectric image contribution to $\Delta F_{\text {sol }}{ }^{*}$ can be neglected. We note later that $\Delta F_{\text {atm }}{ }^{*}$ is apparently much smaller than $\Delta F_{\text {sol }}{ }^{*}$. Dielectric image effects may be estimated from electrostatic calculations to contribute about $8 \%$ to $w^{r}$ when two charges of equal magnitude meet.
}

where $n e$ is $\Delta e$, the charge transferred from one reactant to the other; $a_{1}$ and $a_{2}$ are the radii of the two reactants computed at intramolecular coordinates $q^{i}=q_{*}{ }^{i}$ (the radii are of spheres each of which includes any inner coordination shell); $R$ is taken to be the sum $a_{1}+a_{2}$; $D_{\text {op }}$ is the square of the refractive index of the medium; and $D_{s}$ is the static dielectric constant of the medium

Electrochemical:

$$
\Delta F^{*}{ }_{\mathrm{ool}}=\frac{m^{2}(n e)^{2}}{2}\left(\frac{1}{a_{1}}-\frac{1}{R}\right)\left(\frac{1}{D_{\mathrm{op}}}-\frac{1}{D_{s}}\right),
$$

where $R$ is twice the distance from the center of the ion to the electrode surface and $a_{1}$ is the radius of the reactant (and hence of the product) computed at $q^{i}=q_{*}{ }^{i}$.

The value obtained in Appendix VII for $\Delta F^{*}{ }_{\text {atm }}$ in the electrically unsaturated region (i.e., in the DebyeHückel region for the atmosphere around the ion and, in electrode systems, for the diffuse part of the double layer) is given by Eq. (91) for homogeneous systems for the case of $a_{1}=a_{2}(=a)$, and by $(92)$ for electrode systems. The value for $\Delta F^{*}{ }_{\mathrm{atm}}$ for partially electrically saturated systems can also be obtained from (67). Once again, one introduces "differential" quantities. If the latter are replaced by "mean" values near the central species Eqs. (91) and (92) are again obtained, but with $D_{s}$ and $\boldsymbol{\kappa}$ reinterpreted; $\boldsymbol{\kappa}$ is given by (A23) in Appendix VII. A more reliable procedure, however, would be to use the position-dependent value of $\boldsymbol{\kappa}$ in solving this particular linearized Poisson-Boltzmann equation, since the electric fields in electrolyte media die out fairly rapidly, namely as $r^{-1} \exp (-\boldsymbol{k} r)$. Equations (91) and (92) are based on the solution of a linearized Poisson-Boltzmann equation with a local mean $\mathrm{k}$

Homogeneous:

$$
\begin{aligned}
& \Delta F_{\mathrm{atm}}^{*}=\frac{m^{2}(n e)^{2}}{D_{s} R} \\
& \times\left[\frac{\boldsymbol{\kappa} R+\exp [-\boldsymbol{\kappa}(R-a)]\left(1+\boldsymbol{x}^{2} a^{2} / 2\right)}{1+\boldsymbol{\kappa} a+\exp [-\boldsymbol{\kappa}(R-a)] \boldsymbol{k}^{2} a^{3} / 3 R}-1\right],
\end{aligned}
$$

Electrode:

$$
\Delta F_{\mathrm{atm}}^{*}=\frac{1}{2}[\text { rhs of }(91)] .
$$

Calculated as above, $\Delta F^{*}$ atm is much smaller than $\Delta F^{*}$ sol and is also expected to be less than the salt effects on $w^{r}$ and $w^{p}$. Even at high $\boldsymbol{\kappa}$ it is only $m^{2}(n e)^{2}$ $(R-a) / D_{s} a R$. Since $R \cong 2 a$, its value there is about $m^{2}(n e)^{2} / D_{s} R$, which is only about $2 \%$ of $\Delta F^{*}{ }_{\mathrm{sol}}$. Parenthetically, we note that this term arising from (91) and (92) just cancels the $D_{s}$ term in (89) and (90), respectively.

Added electrolyte can influence the rate constant, we conclude, principally by affecting $w^{r}$, w , and (by affecting dielectric properties) $\Delta F^{*}{ }_{\mathrm{sol}}$.

Comparison of (89) with (90) reveals that $\lambda_{o}$ for an 
isotopic exchange reaction has twice the value of $\lambda_{o}$ for an electrode reaction involving this redox couple when the value of $R$ is the same in each case. It is recalled that $R$ is the value for which $\kappa \rho \exp \left(-\Delta F^{*} / k T\right)$ had a maximum. If one presumes this $R$ to be the distance of closest approach of the "hard spheres" and assumes the reactant to just touch the electrode, then $R$ is the same in each case. In Eq. (89) $a_{1}=a_{2}$ for an isotopic exchange reaction since these are the radii evaluated for $q=q_{*}$, it is recalled, and since typically the transition state should be symmetrical in this respect. (From the equation cited the actual $q *$ 's can be computed and the presumed symmetry verified for typical conditions.)

It may be seen from (89) that $\lambda_{o}$ is essentially equal to the sum of two terms, one per reactant, and that for the same $R$ the value of $\lambda_{0}$ for the homogeneous reaction in any redox system $A$ equals twice its value for the electrochemical case. While the presence of the $R$ term makes $\lambda_{o}$ not quite additive, the deviation from additivity can be shown to be small: On denoting the radii for ions of the two systems by $a$ and $b$ we obtain (93).

$\lambda_{o}^{a b}-\frac{1}{2}\left(\lambda_{o}^{a a}+\lambda_{o}^{b b}\right)=\frac{[1-(b / a)]^{2}}{4 b[1+(b / a)]}(n e)^{2}\left(\frac{1}{D_{\mathrm{op}}}-\frac{1}{D_{3}}\right)$.

Even if $b / a$ is $\frac{1}{2}$, a fairly extreme case, the ratio of the above difference to $\lambda_{0}{ }^{b b}$ is

$$
\frac{(1-b / a)^{2}}{2(1+b / a)}
$$

i.e., $\frac{1}{12}$. In virtue of this result, $\lambda_{o}$ has been treated as an additive function in applications ${ }^{6,7}$ of the equations of this paper.

\section{SIGNIFICANCE OF $m$}

The parameter $m$ was chosen in Sec. 13 so as to satisfy the centering condition (20), a condition which led to the vanishing of $I^{\prime}(0)$. On differentiating $I\left(q^{N}, R\right)$ given in (15) and setting $q^{N}=0$ one finds:

$$
m=-\left\langle\frac{\partial U^{r}}{\partial q^{N}}\right\rangle /\left\langle\frac{\partial}{\partial q^{N}}\left(U^{r}-U^{p}\right)\right\rangle
$$

where the average \langle\rangle is over the distribution function on the reaction hypersurface $S^{\prime}$ at the given $R$,

$$
\exp \left(\frac{-U^{r}}{k T}\right) / \int \exp \left(\frac{-U^{r}}{k T}\right)\left(a^{N N}\right)^{-\frac{1}{2}} d S^{\prime}
$$

From (94), $-m$ is seen to be the mean slope at the reaction hypersurface $S^{\prime},\left\langle\partial U^{r} / \partial q^{N}\right\rangle$, of the $R$ surface, for the given separation distance $R$, divided by the sum of the mean slopes, $\left\langle\partial U^{r} / \partial q^{N}\right\rangle$ and $\left\langle-\partial U^{p} / \partial q^{N}\right\rangle$ of the $R$ and $P$ surfaces at $S^{\prime}$. If the intersection surface $S^{\prime}$ at this $R$ passed through the stable configurations of the reactants, on the average, then $\left\langle\partial U^{r} / \partial q^{N}\right\rangle$ would be zero. If it passed through those of the products instead $\left\langle\partial U^{p} / \partial q^{N}\right\rangle$ would be zero. In these two cases one sees from Eq. (94) that $m$ would be 0 and -1 , respectively. When in the vicinity of $S^{\prime}$ the $R$ and $P$ surfaces are, on the average, mirror images of each other about $S^{\prime}$, $\left\langle\partial U^{r} / \partial q^{N}\right\rangle$ equals $\left\langle-\partial U^{p} / \partial q^{N}\right\rangle$ and one sees that $m=-\frac{1}{2}$. Values of $m$ close to $-\frac{1}{2}$ are typical ${ }^{6,7}$ and are to be expected, one sees from (86), when $\Delta F^{\circ \prime}$ is near zero or when $E$ is close to $E_{o}{ }^{\prime}$ (typically of the order of or less than $10 \mathrm{kcal} \mathrm{mole}{ }^{-1}$ or $0.25 \mathrm{~V}$, respectively).

\section{DEDUCTIONS FROM THE FINAL EQUATIONS}

Equations (31) to (33), together with the additivity property of $\lambda$ (Sec. 15), and the relation between the electrochemical and chemical $\lambda$ 's described earlier lead to the following deductions, if $\kappa$ and $\rho$ are about unity, or if they satisfy milder conditions in some cases. ${ }^{30}$

(a) The rate constant of a homogeneous "cross reaction," $k_{12}$, is related to that of the two electronexchange reactions, $k_{11}$ and $k_{12}$, and to the equilibrium constant $K_{12}$, in the prevailing medium by Eq. (96), when the work terms are small or cancel,

$$
\begin{gathered}
\mathrm{Ox}_{1}+\operatorname{Red}_{2} \stackrel{k_{12}}{\rightleftharpoons} \operatorname{Red}_{1}+\mathrm{Ox}_{2}, \\
k_{12}=\left(k_{11} k_{22} K_{12} f\right)^{\frac{1}{t}},
\end{gathered}
$$

where

$$
\ln f=\frac{\left(\ln K_{12}\right)^{2}}{4 \ln \left(k_{11} k_{22} / Z^{2}\right)} .
$$

Frequently, $f$ is close to unity.

(b) The electrochemical transfer coefficient at metal electrodes is 0.5 for small activation overpotentials ${ }^{31 \mathrm{a}}$ (i.e., if $|n F \eta|<\left|\Delta F_{o}{ }^{*}\right|$, where $\Delta F_{o}{ }^{*}$ is the free energy of activation for the exchange current), ${ }^{31 b}$ when the work terms are negligible.

(c) When a substituent in the coordination shell of a reactant is remote from the central metal atom and is varied in a series, a plot of the free energy of activation $\Delta F^{*}$ versus the "standard" free energy of reaction in the prevailing medium $\Delta F^{\circ \prime}$ will have a slope of 0.5 , if $\Delta F^{\circ \prime}$ is not too large (i.e., if $\left|\Delta F^{\circ \prime}\right|$ is less than the intercept in this plot at $\Delta F^{\circ \prime}=0$ ). In this series, for a sufficiently remote substituent, $\lambda$ and the work terms are constant but $\Delta F^{\circ \prime}$ varies, as in (87a). The slope of the $\Delta F^{*}$-versus- $\Delta F^{\circ \prime}$ plot has been termed the chemical transfer coefficient, ${ }^{6}$ by analogy with the electrochemical terminology.

(d) When a series of reactants is oxidized (reduced) by two different reagents the ratio of the two rate constants is the same for all members of the series in

\footnotetext{
${ }^{30}$ For example, it suffices for some of the deductions that $k \rho$ be constant in a given series of reaction or that it have a geometric mean property.

31 (a) We have phrased this condition for the case that $(\mathrm{Ox})=$ (Red). For any other case, $\eta$ should be replaced by $E-E_{\circ}^{\prime}$. (b) The exchange current cited refers to the value observed when $(\mathrm{Ox})=(\mathrm{Red})$.
} 
the region of chemical transfer coefficients equal to 0.5 [i.e., in the region where $\left|\Delta F^{\circ \prime}\right|\left\langle\left(\Delta F^{*}\right)_{\Delta F_{0}^{\circ}{ }^{\circ}=0}\right.$ in each case].

(e) When the series of reactants in (d) is oxidized (reduced) electrochemically at a given metal-solution potential difference the ratio of the electrochemical rate constant to either of the chemical rate constants in (d) is the same for all members of the series, in the region where the chemical and (work-corrected) electrochemical transfer coefficient is 0.5 .

(f) The rate constant of a (chemical) electronexchange reaction, $k_{\mathrm{ex}}$, is related to the electrochemical rate constant at zero activation overpotential, ${ }^{, 1 a} k_{\mathrm{el}}$, for this redox system, according to Eq. (98) when the work terms are negligible:

$$
\left(k_{\mathrm{ex}} / Z_{\mathrm{soln}}\right) \stackrel{\mathfrak{l}}{\cong} k_{\mathrm{el}} / Z_{\mathrm{el}}
$$

where $Z_{\text {soln }}$ and $Z_{\mathrm{el}}$ are collision frequencies, namely about $10^{11} \mathrm{~mole}^{-1} \cdot \mathrm{sec}^{-1}$ and $10^{4} \mathrm{~cm} \mathrm{sec}^{-1}$.

When the work terms are not negligible, or do not cancel in the comparison, the deductions which depend on this condition refer to rate constants, to $K_{12}$ and to an electrochemical transfer coefficient corrected for these terms. Again, a minor modification of the transfer coefficients from the value of $\frac{1}{2}$ in (b) or (c) can also arise from the antisymmetrical force constant term $\left\langle l_{s}\right\rangle$ in Eqs. (87) and (A14).

It is shown in Appendix VII that under certain conditions these expected correlations apply to over-all rate constants as well as to those involving only one pair of reactants.

\section{GENERALIZATION AND OTHER IMPROVEMENTS}

Some of the extensions or improvements in the present paper, compared with the earlier ones in this series, are the following:

(1) Use is made of a more general expression for the reaction rate as the starting point.

(2) A more detailed picture of the mechanism of electrode transfer is given for the electrochemical case.

(3) The derivation is now given for both electrode and homogeneous reactions, and in a single treatment.

(4) The statistical-mechanical treatment of polar interactions, based in Part IV on the interactions of permanent and induced dipolar molecules in the medium, was replaced by a more general particle description of polar interactions, through the use of the potential-energy function (37) and (65).

(5) The equivalent equilibrium distribution made plausible in Part IV was proved more rigorously here.

(6) The functional form (68) for $\Delta F_{o}^{*}$, obtained in Part IV only by subsequently treating the medium as a dielectric continuum, was derived here using a statistical-mechanical treatment of nonequilibrium polarization systems.
(7) The basic equation for $k_{\text {rate }}$ has been converted to a simple form [e.g., (31) and (81)], a form used in Part $\mathrm{V}$, by neglecting the antisymmetrical function of the force constants, a neglect which has only a minor effect numerically.

(8) The symmetry arguments used in Part IV to convert the $k T / h$ and a portion of a $\Delta F^{*}$ term to the $Z$ factor in (31) have now been given more rigorously.

(9) The ion atmospheric reorganization term was but mentioned in Part IV. It is now incorporated into $\Delta F_{o}{ }^{*}$. The nonpolar contribution to $w^{r}$ and $w^{p}$ is also formally included.

(10) The contribution of a range of separation distances to the rate constant is included.

The results in the present paper may be compared with earlier papers in this series. In Part I, $\Delta F_{o}{ }^{*}$ was computed for homogeneous reactions at zero ionic strength, and dielectric continuum theory was used. Equation (89) was obtained. The actual mechanism of electron transfer was discussed there, but without the detailed description which the use of many-dimensional potential energy surfaces provides. The latter was used in later papers of this series, a use which added to the physical picture. The counterpart of Part I for electrode systems was also derived and applied to the data in a subsequent paper. ${ }^{2}$

In the earliest papers, the dielectric continuum equivalent of the equivalent equilibrium distribution was derived by a method apparently different from that used in the present paper. The distribution selected was the one which minimized the free energy subject to the constraint embodied in Eq. (20), or really embodied in the dielectric continuum counterpart of (20). In Appendix IX this method is in fact shown to yield the same result for the equivalent equilibrium distribution as the functional analytic one used in Appendix II. It is entirely equivalent.

\section{APPENDIX I. NONADIABATIC ELECTRON TRANSFERS}

Several estimates are available for the probability of nonadiabatic reactions, per passage through the intersection region of two potential energy surfaces, and have been referred to and discussed in Ref. 7 . In each case the motion along the reaction coordinate was assumed to be dynamically separable from the remaining motions. (For conditions on separability see, for example, Ref. 32 and references cited therein.) The probability of electron transfer per passage through the intersection region in Fig. 1 will depend in the first approximation on the momentum $p_{N}$ conjugate to the reaction coordinate $q^{N}$, as, for example, in the LandauZener ${ }^{11}$ equation. While the value of $\kappa$ is not so simply represented in more rigorous treatments, we simply write it as $\kappa\left(p_{N}\right)$. In the above treatments the reaction coordinate was assumed to be orthogonal to the others

${ }_{32}$ R. A. Marcus, J. Chem. Phys. 41, 603 (1964). 
in mass-weighted configuration space, so that $g_{N i}$ vanishes for $i \neq N$ (and so, therefore, does $g^{N i}$ ) in the kinetic energy. On recalling the derivation of Equations (1) and $(2)^{8}$ and on introducing the above assumptions, the rate constant is given, one can show, by Eqs. (1) or (2), but with the integrand multiplied by $\kappa$ :

$$
\kappa=\frac{\int_{p_{N}=0}^{\infty} \kappa\left(p_{N}\right) g^{N N} p_{N} \exp \left(-\frac{g^{N N} p_{N}{ }^{2}}{2 k T}\right) d p_{N}}{\int_{0}^{\infty} g^{N N} p_{N} \exp \left(-\frac{g^{N N} p_{N}{ }^{2}}{2 k T}\right) d p_{N}} .
$$

This $\kappa$ can depend on all the other coordinates, $q^{i}(i \neq N)$ at the given value of $q^{N}$ characterizing the intersection surface $S$. The denominator in the above equation is easily shown to equal $k T$, and so to be independent of the $q^{i}$. In the discussions of $\kappa\left(p_{N}\right)$ in the literature, the derivation of the Landau-Zener equation, for example, the reaction coordinate has been assumed to be rectilinear; $g^{N N}$ is then a constant and the integral in the numerator then becomes independent of the $q^{i}$ and may be removed from the integral in Eqs. (1) and (2).

There appears to be no treatment in the literature for nonadiabatic reactions involving many closely spaced energy surfaces as in Fig. 2, covering the range of $\kappa\left(p_{N}\right)$ from 0 to 1 . If $k\left(p_{N}\right)$ is sufficiently small, the transition to each $P$ surface from the initial $R$ surface may be assumed to be independent, as mentioned earlier, and the reverse transition to the initial $R$ surface during this passage may also be neglected. In this case only does the method of Levich and co-workers in this connection become appropriate. (For references see Ref. 7.) In this case the above $\kappa$ appears in the integrand of Eq. (3) and care is taken to sum over all levels in an appropriate fashion, as done by Levich et al. (see Ref. 7 for bibliography). One can then evaluate the $\kappa$ appearing in Eq. (33) and defined earlier. Usually, however, we assume that $\kappa\left(p_{N}\right)$ is close to unity (within some small numerical factor, say) for the $p_{N}$ 's of interest.

\section{APPENDIX II. PROOF OF EQ. (13) FOR THE CENTERED DISTRIBUTION}

The centering is of both a horizontal type (horizontal in terms of Fig. 1 or 2) and of a vertical type, represented by Eqs. (A1) and (A2), respectively:

$$
\begin{aligned}
& \int f^{*} U^{r} d V^{\prime}=\int f^{*} U^{p} d V^{\prime}, \\
& \int f^{*} U^{*} d V^{\prime}=\int f^{*} U^{r} d V^{\prime} .
\end{aligned}
$$

Suppose, for possibly more general applications, that there are $n$ linear equations of constraint of the type represented by (A3). Here, we are especially interested in the case $n=1$,

$$
\int f^{*} y_{j} d V^{\prime}=0, \quad j=1, \cdots, n .
$$

For any temperature and $U^{*}$, this integral is a linear functional of $y_{j}$. Although one can find functions, $u$, other than $y_{j}$ (and other than linear combinations of $y_{j}$ ) for which $\int f^{*} u d V^{\prime}$ vanishes at some temperature $T$, the $y_{j}$ 's are the only ones for which this integral is specified to vanish for all $T$. That is, there are only the $n$ equations of constraint (A3) on the $U^{*}$ in $f^{*}$. The space functions $Y$ for which $\int f^{*} Y d V^{\prime}$ is real and finite form a linear vector space over the field of real scalars. Moreover, the integral, denoted by $J(Y)$, is a linear functional on this space. For some subspace $M$ of it, the integral vanishes. The functions $y_{j}(j=1$ to $n)$ form a basis for $M$. If there exists some function $w$ for which $J(w)$ does not vanish, then an elementary theorem ${ }^{33}$ of functional analysis shows that any function $x$ can be written as

$$
x=w[J(x) / J(w)]+y,
$$

where $y$ belongs to $M$. In the present instance $w=1$ is such a function. On applying (A4) to the function $x=U^{*}-U^{r}$ and using (A2) one sees that $x=y$, i.e., that $x$ belongs to $M$ and can so be written as a linear combination of the functions $y_{j}$. In the present case, $M$ is one dimensional, the only $y_{j}$ being $U^{r}-U^{p}$, since (A1) is the only equation of constraint. Thus, $x$, i.e., $U^{*}-U^{r}$, equals $U^{r}-U^{p}$ multiplied by a real scalar, and Eq. (13) is established.

\section{APPENDIX III. DISTRIBUTION OF $V_{o}^{\prime}$ COORDI- NATES IN THE ACTIVATED COMPLEX}

We first note that $U(2)$ in Eq. (65) does not depend on $\rho_{M}{ }^{\circ}$, the $\rho^{\circ}$ of the "medium," and so is insensitive to the usual rotational and translational fluctuations of the solvent molecules, unlike $U(0)$ and $U(1)$. Since $U_{o}{ }^{*}$ is given by (13), with $o$ subscripts added, one term in $U_{o}^{*}$ is $U^{r}(2)+m\left[U^{r}(2)-U^{p}(2)\right]$. Since this can be extracted from the integral in the denominator of the above distribution function because of this insensitivity to the $V^{\prime}{ }_{0}$ coordinates, it cancels a corresponding term extracted from the numerator. The distribution function $f_{0}^{*}$ then becomes (A5) :

$$
\frac{\exp \left(-\left\{U(0)+U^{r}(1)+m\left[U^{r}(1)-U^{p}(1)\right]\right\} / k T\right)}{\int \exp \left(-\left\{U(0)+U^{r}(1)+m\left[U^{r}(1)-U^{p}(1)\right]\right\} / k T\right) d V^{\prime}{ }_{o}}
$$

Since $U(1)$ is linearly dependent on the $\rho_{a}{ }^{\circ}$ of each reactant, $U^{r}(\mathbf{1})+m\left[U^{r}(\mathbf{1})-U^{p}(1)\right]$ equals the $U(1)$ for a system in which each reactant has a $\rho_{a}{ }^{\circ}, \rho_{a}{ }^{0 *}$,

\footnotetext{
${ }^{33}$ A. E. Taylor, Introduction to Functional Analysis (John Wiley \& Sons, Inc. New York, 1958), p. 138.
} 
given by (66). Next, on multiplying the numerator and denominator of (A5) by the exponential of the $-U(2) / k T$ corresponding to these $\rho_{a}{ }^{\circ *}$ 's and placing it under the integral sign, we see that the distribution function $f_{o}^{*}$ is the same as that corresponding to the $\rho_{a}{ }^{\circ *}$ 's given by (66).

\section{APPENDIX IV. SIMPLIFICATION OF EQ. (54) AND THE EQUATION FOR $k_{\text {rate }}$}

We introduce the quantities $k_{j k}$ and $l_{j k}$ defined in (63) and (64). The first was chosen so as to have dimensions of a force constant, and the second so as to be dimensionless.

Principally, it is the diagonal stretching contributions which are usually important. Purely for simplicity of argument we confine our attention to the diagonal terms. We denote the new force constants by $f_{s}{ }^{r}, f_{8}{ }^{p}$, and their symmetric and antisymmetric combinations cited above by $k_{s}$ and $l_{s}$. In terms of $k_{s}$ and $l_{s}, f_{s}{ }^{r}$ equals $k_{s} /\left(1-l_{s}\right)$ and $f_{s}{ }^{p}$ equals $k_{s} /\left(1+l_{s}\right)$. To make use of the symmetry of the resulting equations we use the parameter $\epsilon$, equal to $\left(m+\frac{1}{2}\right)$.

We obtain (A6) from (54) and (60):

$$
\begin{aligned}
\Delta F_{i}^{*}=\frac{1}{2}\left(\epsilon-\frac{1}{2}\right)^{2} & \sum_{s} k_{s}\left(\Delta q_{s}{ }^{\circ}\right)^{2}\left(1-l_{s}\right)\left(1+2 \epsilon l_{s}\right)^{-2} \\
& +\frac{1}{2} k T \sum_{s} \ln \left[\left(1+2 \epsilon l_{s}\right) /\left(1+l_{s}\right)\right],
\end{aligned}
$$

where $\Delta F_{i}{ }^{*}$ is defined as $\Delta F^{*}(R)-\Delta F_{o}{ }^{*}(R)$. Similarly, we find (A7) by noting that it is obtained from (A6) by replacing $-m$ by $m+1$ and interchanging $r$ and $p$ subscripts (see Sec. 13)

$$
\begin{array}{r}
\Delta F_{i}^{* p}=\frac{1}{2}\left(\epsilon+\frac{1}{2}\right)^{2} \sum_{s} k_{s}\left(\Delta q_{s}^{\circ}\right)^{2}\left(1+l_{s}\right)\left(1+2 \epsilon l_{s}\right)^{-2} \\
+\frac{1}{2} k T \sum_{s} \ln \left[\left(1+2 \epsilon l_{s}\right) /\left(1-l_{s}\right)\right],
\end{array}
$$

where $\Delta F_{i}{ }^{* p}$ is $\Delta F^{* p}(R)-\Delta F_{o}^{* p}(R)$.

In terms of $\epsilon$, Eq. (79) can be written as (A8), upon introducing $\mathrm{Eq}$. (68) for $\Delta F_{o}{ }^{*}$ and its counterpart for $\Delta F_{o}{ }^{*}\left[=(m+1)^{2} \lambda_{o}\right]$

$$
-2 \epsilon \lambda_{0}+\Delta F_{i}^{*}-\Delta F_{i}^{* p}=\Delta F_{R}{ }^{\circ},
$$

where

$$
\Delta F_{R}{ }^{\circ \prime}=\Delta F^{\circ \prime}+w^{p}-w^{r} .
$$

Most of the data are obtained in the vicinity of $\Delta F_{R}{ }^{\circ \prime}=0 .^{6,7}$ We consider this region first. Near the point $\left(l_{s}=0, \Delta F_{R}{ }^{\circ \prime}=0\right)$ one readily verifies from the equations below that $\epsilon$ is close to zero and that it vanishes at that point. We let $\eta$ denote $\epsilon$ or $l_{s}$, and $O$ denote the "order of." ( $\eta$ is a small quantity in the vicinity of this point.) One then finds from (A6) to (A8)

$$
-2 \epsilon \lambda-\frac{1}{2} \lambda_{i}\left\langle l_{s}\right\rangle+O\left(\eta^{3}\right)=\Delta F_{R}{ }^{\circ \prime}+k T \sum_{s} l_{s},
$$

where

$$
\begin{aligned}
\lambda_{i} & =\frac{1}{2} \sum_{s} k_{s}\left(\Delta q_{s}^{\circ}\right)^{2}, \quad \lambda=\lambda_{o}+\lambda_{i}, \\
\left\langle l_{s}\right\rangle & =\sum_{s} k_{s}\left(\Delta q_{s}^{\circ}\right)^{2} l_{s} / \sum_{B} k_{s}\left(\Delta q_{s}^{\circ}\right)^{2} .
\end{aligned}
$$

Furthermore, according to (79) $\Delta F^{*}$ equals $\Delta F^{* p}+$ $\Delta F_{R}{ }^{\circ \prime}$. Hence,

$$
\Delta F^{*}=\frac{1}{2}\left(\Delta F^{*}+\Delta F^{*}\right)=\frac{1}{2}\left(\Delta F^{*}+\Delta F^{* p}\right)+\frac{1}{2} \Delta F_{R}{ }^{\circ \prime} .
$$

On introducing (A6) and (A 7$)$ one finds

$\Delta F^{*}=\frac{1}{2} \Delta F_{R}{ }^{\circ \prime}+\lambda\left(\epsilon^{2}+\frac{1}{4}\right)+\lambda_{i} O\left(\eta^{4}\right)+\frac{1}{4} k T \sum_{s}\left(4 \epsilon l_{s}+l_{s}^{2}\right)$.

On introducing (A10) for $\epsilon$ one finds that (A12) becomes

$$
\begin{aligned}
\Delta F^{*}= & \frac{1}{2} \Delta F_{R}{ }^{\circ \prime}+\frac{1}{4} \lambda+(1 / 4 \lambda)\left(\Delta F_{R}{ }^{\circ}+\frac{1}{2} \lambda_{i}\left\langle l_{s}\right\rangle\right)^{2} \\
& +\frac{1}{4} k T\left[\sum_{s} l_{s}^{2}-(k T / \lambda)\left(\sum_{s} l_{s}\right)^{2}\right]+O\left(\eta^{4}\right) .
\end{aligned}
$$

The same expression obtains for electrode processes, with the $\Delta F^{\circ \prime}$ in $\Delta F_{R}{ }^{\circ \prime}$ replaced by $n e\left(E-E^{0^{\prime}}\right)$.

In an isotopic exchange reaction which involves mere interchange of charges in the electron transfer step, the term $\left\langle l_{s}\right\rangle$ vanishes by symmetry. In other reactions there will be some tendency for it to vanish, for while $l_{s}$ increases on one reactant on going from State $R$ to State $P$ (due to an increase of charge), it will tend to decrease on the other. As a somewhat extreme case involving no compensation, consider two reactants one of which has vanishing $l_{s}$ and also vanishing contribution to $\lambda_{i}$. (Hence, we include the possibility that this "reactant" is an electrode.) For the other molecule let the force constants $k_{8}{ }^{r}$ and $k_{8}{ }^{p}$ differ by as much as a factor of 2 . Then one finds $\left\langle l_{s}\right\rangle \sim \frac{1}{3}$. If $\lambda_{i} / \lambda \sim \frac{1}{2}$ then $\lambda_{i}{ }^{2}\left\langle l_{s}\right\rangle^{2} / 16 \lambda$ is only about $1 \%$ of $\lambda / 4$, the main term at $\Delta F_{R}{ }^{\prime \prime}=0$. When $\lambda / 4$ has its usual value of 10 to 20 $\mathrm{kcal} \mathrm{mole}^{-1}$, say 10 , and when the reactant has a coordination number of six, then the $k T$ term in (A13), is estimated to be about $4 \%$ of the $\lambda / 4$ term at room temperature.

We consider next the effect of nonvanishing $\left\langle l_{s}\right\rangle$ on the derivative $\left(\partial \Delta F^{r} / \partial \Delta F^{\circ \prime}\right)_{\lambda_{0}, \lambda_{i}}$ at $\Delta F_{R}{ }^{\circ \prime}=0$, the region of greatest interest. This derivative equals

$$
\frac{1}{2}+\left(\lambda_{i} / 4 \lambda\right)\left\langle l_{s}\right\rangle+O\left(\eta^{2}\right) .
$$

In the case cited above the $\lambda_{i}\left\langle l_{s}\right\rangle / 4 \lambda$ term is about +0.04 . Thus, the derivative differs by only $8 \%$ for this case. Hence, the $\left\langle l_{s}\right\rangle$ term may be neglected when $\epsilon$ (and hence $\left|\Delta F_{R}{ }^{\circ \prime} / \lambda\right|$ ) is small. When $\left|\Delta F_{R}{ }^{\circ \prime} / \lambda\right|$ is not small, one finds that (A13) should be replaced by (A14a), to terms correct to first order in the $l_{\mathrm{s}}$

$$
\begin{aligned}
& \Delta F^{*}=\frac{1}{2} \Delta F_{R}{ }^{\circ \prime}+\frac{1}{4} \lambda+\frac{1}{4}\left(\Delta F_{R}{ }^{\circ \prime}\right)^{2} \\
& +\left(\Delta F_{R}{ }^{\prime \prime} / 4 \lambda\right) \lambda_{i}\left\langle l_{s}\right\rangle\left[1-\left(\Delta F_{R}{ }^{\circ \prime} / \lambda\right)^{2}\right] .
\end{aligned}
$$


The term containing $\left\langle l_{s}\right\rangle$ is still small: A fairly extreme case is one where the activated complex resembles the reactants $(m=0)$ or the products $(m=1)$. At each extreme $\left|\Delta F_{R}{ }^{\circ \prime} / \lambda\right|$ is about unity, since the expression for $\epsilon\left(=-\Delta F_{R}{ }^{\circ} / 2 \lambda\right)$ is but slightly affected and since $|\epsilon|$ equals $\frac{1}{2}$ when $m$ is 0 or 1 . In the interval

$$
0 \leq\left|\Delta F_{R}{ }^{\circ} / \lambda\right| \leq 1
$$

the last term in (A14a) has a maximum at

$$
\left|\Delta F_{R}{ }^{\prime \prime} / \lambda\right|=\left(\frac{1}{3}\right)^{\frac{1}{2}} \text {. }
$$

At this point it equals about $1 \mathrm{kcal} \mathrm{mole}{ }^{-1}$ for the values of $\left\langle l_{s}\right\rangle, \lambda_{i} / \lambda$, and $\lambda / 4$ cited above. When one does not neglect second and higher-order terms in $l_{s}$, and solves (A6) to (A8) numerically in this region one obtains the same result: The $l_{s}$ terms may be neglected.

APPENDIX V. SMALLNESS OF $\left\langle U^{r}\left(\mathbf{Q}_{*}\right)-U^{p}\left(\mathbf{Q}_{*}\right)\right\rangle_{* \circ}$

If $\left\langle U^{r}-U^{p}\right\rangle_{*_{o}}$ at any $\mathbf{Q}$ is expanded about its value at $\mathbf{Q}_{*}$ and if it can be shown that the linear term suffices, it follows that $\left\langle U^{r}-U^{p}\right\rangle_{*_{o}}$ averaged over $f_{i}{ }^{*}$ equals the value at $\mathbf{Q}$ * plus the average of the linear term. In virtue of $(50)$ the averaged linear term vanishes and, in virtue of $(20)$, the average of $\left\langle U^{r}-U^{p}\right\rangle_{* o}$ over $f_{i}{ }^{*}$ vanishes. Hence, $\left\langle U^{r}\left(\mathbf{Q}_{*}\right)-U^{p}(\mathbf{Q} *)\right\rangle *_{o}$ also vanishes.

To show by a posteriori calculation that the linear term in the expansion suffices we make use of some notation introduced after (54). After use of (37), of the equality of $\left\langle U_{o}^{r}-U_{o}^{p}\right\rangle_{*_{o}}$ with $F_{o}{ }^{*}-F_{o}{ }^{* p}$, of (68), of the definition of $\bar{U}_{i}^{r}$ and $\vec{U}_{i}^{p}$, and of their quadratic expansions about $\mathbf{Q}_{r}$ and $\mathbf{Q}_{p}$, respectively, of the essential equality of the vibrational entropy of reactants and products, and of the justifiable neglect of the antisymmetrical functions (64) (Appendix IV) one finds (A15) for any given $\mathbf{Q}$.

$$
\begin{aligned}
\left\langle U^{r}-U^{p}\right\rangle_{*_{o}}=-(2 & +1) \lambda_{o}(\mathbf{Q})-\Delta F^{\circ \prime} \\
& +\frac{1}{2} \sum_{i, j} k_{i j}\left(q^{i}-q_{r}^{i}\right)\left(q^{j}-q_{r}^{j}\right) \\
& -\frac{1}{2} \sum_{i, j} k_{i j}\left(q^{i}-q_{p}^{i}\right)\left(q^{j}-q_{p}^{j}\right) .
\end{aligned}
$$

The quadratic term, $k_{i j} q^{i} q^{j}$, is seen to cancel. A linear expansion of $\lambda_{o}(Q)$ about $\lambda_{o}\left(Q^{*}\right)$ is sufficient, for even the linear term is small (compare Appendix VI). Hence, he linear term in an expansion of $\left\langle U^{r}(\mathbf{Q})-U^{p}(\mathbf{Q})\right\rangle_{*}$ suffices. The vanishing of $\left\langle U^{r}\left(\mathbf{Q}_{*}\right)-U^{p}\left(\mathbf{Q}_{*}\right)\right\rangle_{*_{o}}$ then follows.

\section{APPENDIX VI. JUSTIFICATION OF NEGLECT OF $\partial \lambda_{\circ} / \partial q^{i}$ IN THE DERIVATION OF EQ. (58)}

It is shown here that the error in neglecting the dependence of $\lambda_{o}$ on $\mathbf{Q}$ in deducing (58) from (56) is minor.

Since the arguments in Appendix IV reveal that the error in neglecting the antisymmetrical functions (64) is minor, we may simplify the present analysis by neglecting them. To this purpose all force constants may be replaced by the symmetrical ones, $k_{j k}$, defined by Eq. (63).

Let $\boldsymbol{\Lambda}_{o}$ be a column matrix whose components are $\partial \lambda_{o} / \partial q^{i}$ :

$$
\begin{aligned}
\lambda_{o}(\mathbf{Q}) & =\lambda_{o}\left(\mathbf{Q}_{*}\right)+\sum_{i}\left(\partial \lambda_{o} / \partial q^{i}\right)\left(q^{i}-q *^{i}\right)+\cdots \\
& =\lambda_{o}(\mathbf{Q} *)+\mathbf{\Lambda}_{o}{ }^{T} \cdot(\mathbf{Q}-\mathbf{Q} *)+\cdots
\end{aligned}
$$

The first variation in an expansion of $\bar{U}_{i}^{*}(\mathbf{Q})$ about $Q *$ is found from (56)

$$
\begin{aligned}
\delta \bar{U}_{i}{ }^{*}=\delta \mathbf{Q}^{T} & {\left[(m+1) \mathbf{K}\left(\mathbf{Q}_{*}-\mathbf{Q}_{r}\right)\right.} \\
& \left.-m \mathbf{K}\left(\mathbf{Q}_{*}-\mathbf{Q}_{p}\right)-m(m+1) \mathbf{\Lambda}_{o}\right],
\end{aligned}
$$

where the elements of $\mathbf{K}$ are the $k_{j k}$ 's.

On setting $\delta \bar{U}_{i}^{*}$ equal to zero, one obtains, instead of (58);

$$
\mathbf{Q}_{*}=m(m+1) \mathbf{K}^{-1} \mathbf{\Lambda}_{o}+(m+1) \mathbf{Q}_{r}-m \mathbf{Q}_{p} .
$$

Equation (54) for $\Delta F^{*}$ then becomes

$$
\begin{aligned}
\Delta F^{*}=\left(m^{2} / 2\right) & {\left[\Delta \mathbf{Q}^{T}+(m+1)\left(\mathbf{K}^{-1} \mathbf{\Lambda}_{o}\right)^{T}\right] } \\
& \cdot K\left[\Delta \mathbf{Q}^{T}+(m+1) \mathbf{K}^{-1} \mathbf{\Lambda}_{o}\right] \\
& +m^{2} \lambda_{o}\left(Q^{*}\right)+\frac{1}{2} k T \ln \left|f_{j k}{ }^{*}\right| /\left|k_{j k}\right| .
\end{aligned}
$$

For present purposes it suffices to consider the case where $\Delta F^{\circ}$ is small. An expression for $\Delta F^{* p}$ can be obtained from (A19) by replacing $m$ by $-(m+1)$ and $\Delta \mathbf{Q}$ by $-\Delta \mathbf{Q}$. On letting $\Delta F^{*}-\Delta F^{* p}$ equal zero (since $\Delta F^{\circ \prime}$ is zero) the resulting equation is solved for $m$, which is thereby found to be $-\frac{1}{2}$. A simple numerical estimate then shows that the presence of the $\mathbf{K}^{-1} \mathbf{\Lambda}_{o}$ terms have negligible effect: Other than the in term the rhs of (A19) is given by

$$
\frac{1}{4} \lambda\left(\mathbf{Q}_{*}\right)+\frac{1}{4} \Delta \lambda_{o}+\frac{1}{32} \boldsymbol{\Lambda}_{o}^{T}\left(\mathbf{K}^{T}\right)^{-1} \cdot \boldsymbol{\Lambda}_{o},
$$

where $\Delta \lambda_{o}$ is the total change in $\lambda_{o}$ when $Q_{r}$ is changed to $Q_{p}$. Typically $\lambda_{o} / 4$ is of the order of $5 \mathrm{kcal} \mathrm{mole}^{-1}$ and is inversely proportional to ion size. When the mean bond length changes by as much as $0.15 \mathrm{~A}$ (compare the probable $\mathrm{Fe}-\mathrm{O}$ bond length difference in $\mathrm{Fe}^{2+}$ and $\mathrm{Fe}^{3+}$ hydrates) and when the radius of the reactant including inner coordination shell is $3 \mathrm{~A}, \Delta \mathrm{\lambda}_{o} / 4$ is about $\frac{1}{5}(0.15 / 3)$, i.e., about $0.25 \mathrm{kcal} \mathrm{mole}^{-1}$. The third term in (A20) is even less. For example, if one considers the stretching of bonds only, and if the stretching $k_{j}$ 's for metal-oxygen bonds in a hydrated cation are taken to be the same one finds

$$
\frac{1}{32} \Lambda_{o}^{T}\left(K^{T}\right)^{-1} \Lambda_{o}=\left(\Delta \lambda_{o} / \lambda_{i}\right)^{2} \frac{1}{4} \lambda_{i} .
$$

(Similar remarks apply to other coordination complexes.) Since $\lambda_{i} / 4$ is of the order of $10 \mathrm{kcal} \mathrm{mole}^{-1}$ for the cited case (A21) is about $0.006 \mathrm{kcal} \mathrm{mole}^{-1}$.

\section{APPENDIX VII. CALCULATION OF $\triangle F_{o}{ }^{*}$ IN CONTINUUM APPROXIMATION}

When dielectric unsaturation and electric unsaturation prevail there is, respectively, a linear response of 
the solvent polarization and of the charge density of ions in an ion atmosphere to the charging of the central ion (or ions), and not merely to a small change in its charge. In real systems, some partial dielectric saturation outside of the coordination shells may occur and, at appreciable concentration of added electrolyte, the response of the atmospheric ions is certainly nonlinear. (The region of linear response of an ion atmosphere to a charging of the "central ion" is confined to the Debye-Hückel region.)

We introduce the partial saturation approximation, wherein only a linear response to a small change in charge of the central ion (ions) is assumed. The special case of unsaturation is automatically included, therefore. We are interested, typically, in changes of magnitude, $m \Delta e$, i.e., about $\frac{1}{2}$ an electronic charge unit. Equation (67) was derived for both the partially saturated and for unsaturated systems, but in the former case the definition of $F^{\mathrm{op}_{m(r-p)}}$ and $F_{m(r-p)}$ has to be interpreted carefully.

To calculate $F_{m(r-p)}$ appearing in (67) and to take partial saturation into account, one considers two charge distributions: (i) The original charge distribution of the reactants and the medium for the cited $R$. (ii) A hypothetical charge distribution in which the reactants' charge distribution is altered from (i) by an amount $m\left(\rho_{a r}{ }^{\circ}-\rho_{a p}{ }^{\circ}\right)$, in a hypothetical system which has responded linearly to this change. To obtain the properties of the hypothetical system in $F_{m(r-p)}$ one substracts the above two charge distributions on the reactants and also substracts the portions of the remaining charge distributions, induced or otherwise, which did not respond. One now has in this hypothetical $[m(r-p)]$ system reactants which have permanent charges given by the distribution $m\left(\rho_{a r}{ }^{\circ}-\rho_{a p}{ }^{\circ}\right)$ and are imbedded in a medium of solvent and atmospheric ions which has linear "response functions" describing the above response. For example, if we use a continuum model, then the effective dielectric susceptibility of the solvent is the proportionality constant $\chi(\mathbf{r})$ in $^{34}$

$$
\delta \mathbf{P}(\mathbf{r})=-\chi(\mathbf{r}) \delta \mathbf{E}(\mathbf{r}),
$$

where $\delta \mathbf{P}$ and $\delta \mathbf{E}$ are the change in polarization and in electric field at $\mathbf{r}$. The effective dielectric constant describing the response to this $\delta \mathbf{E}$ is $D_{s}(\mathbf{r})$ equal to $1+4 \pi \chi(\mathbf{r})$. The quantities $\chi(\mathbf{r})$ and $D_{s}(\mathbf{r})$ can be tensors.

Then, again, if $\rho(\mathbf{r})$ is the charge distribution in the ion atmosphere and, if one wishes, in the electrical double layer at the electrode-solution interface, and if $\rho(\mathbf{r})$ is approximated by a continuum expression

$$
\rho(\mathbf{r})=\sum_{i} c_{i} e_{i} \exp \left(-e_{i} \psi / k T\right),
$$

where $e_{i}$ is the charge of Species $i$ in this atmosphere,

${ }^{34}$ R. A. Marcus, J. Chem. Phys. 38, 1858 (1963). $c_{i}^{\infty}$ is its concentration at infinity, and $\psi$ is the potential at $\mathbf{r}$ relative to the value at infinity, then

$$
\delta \rho(\mathbf{r})=-\left(\sum_{i} c_{i} e_{i} e^{2} e^{-e_{i} \psi / k T} / k T\right) \delta \psi(\mathbf{r}) .
$$

On recalling that the Debye kappa is defined as the square root of the proportionality constant of $\rho(\mathbf{r})$ and $-\psi(\mathbf{r})$ in linear systems, the quantity which plays the same role in this hypothetical system is $\boldsymbol{\kappa}^{\prime}(\mathbf{r})$.

$$
\boldsymbol{\kappa}^{\prime}(\mathbf{r})=\left[\sum_{i} c_{i}{ }^{\infty} e_{i}^{2} \exp \left(-e_{i} \psi / k T\right)\right]^{\frac{1}{2}}
$$

To calculate $F^{\mathrm{op}_{m(r-p)}}$ we recall that this system responds only via the electronic polarization of the medium, and so $\boldsymbol{x}^{\prime}$ vanishes for this system and $\chi^{\prime}(\mathbf{r})$ becomes $\chi_{e}^{\prime}(\mathbf{r})$, the proportionality constant replacing $\chi(\mathbf{r})$ in (A22). The medium in this hypothetical system behaves as though it had a dielectric constant $D^{\prime}{ }_{\text {op }}(\mathbf{r})$ equal to $1+4 \pi \chi_{e}$.

If we take $D_{\text {op }}^{\prime}$ to be approximately a constant, for simplicity, then $F^{o p_{(r-p)}}$ is easily calculated. We neglect dielectric image effects. ${ }^{29} F^{\mathrm{op}} \mathrm{p}_{m(r-p)}$ is the sum of the free energy of solvation of the central species when they are far apart, plus the free energy change when they are brought together in this "op" medium. The former is given by the Born formula (it is not the free energy of solvation of the bare ion, but of the coordinated ion) and the latter by the Coulombic term. Hence,

$$
\begin{array}{r}
F_{m(r-p)}^{\mathrm{op}}=-\left[\frac{(m \Delta e)^{2}}{2 a_{1}}\left(1-\frac{1}{D_{\mathrm{op}}^{\prime}}\right)+\frac{(m \Delta e)^{2}}{2 a_{2}}\left(1-\frac{1}{D^{\prime}{ }_{\mathrm{op}}}\right)\right] \\
-\frac{(m \Delta e)^{2}}{D_{\mathrm{op}}^{\prime} R} .
\end{array}
$$

The $F_{m(r-p)}$ term is the sum of its value when the ion atmosphere does not respond

$$
-\left[\frac{(m \Delta e)^{2}}{2 a_{1}}\left(1-\frac{1}{D^{\prime}}\right)+\frac{m \Delta e}{2 a_{2}}\left(1-\frac{1}{D_{s}^{\prime}}\right)\right]-\frac{(m \Delta e)^{2}}{D_{s}^{\prime} R},
$$

and the contribution due to their response via $\boldsymbol{k}^{\prime}(\mathbf{r})$, $\Delta F^{*}{ }_{\text {atm }}$. On taking $\boldsymbol{x}^{\prime}$ to be approximately a constant near the central series the leading terms of the second contribution $\operatorname{are}^{35}$

$$
-\frac{(m \Delta e)^{2}}{D_{8}^{\prime} R}\left[\frac{\boldsymbol{k}^{\prime} R+\exp \left[-\boldsymbol{k}^{\prime}(R-a)\right]\left(1+\kappa^{\prime 2} a^{2} / 2\right)}{1+\kappa^{\prime} a+\exp \left[-\boldsymbol{\kappa}^{\prime}(R-a)\right] \boldsymbol{\kappa}^{\prime 2} a^{3} / 3 R}-1\right]
$$

when $a_{1}=a_{2}$.

The difference of (A24) and (A25) is the value of $F^{\mathrm{op}}-F$ when the atmosphere does not respond, and

\footnotetext{
${ }^{35}$ Since dielectric image effects are being neglected one may merely use the expressions obtained by G. Scatchard and J. G. Kirkwood, Physik. Z. 33, 297 (1932), for the contribution to the free energy of interaction of a pair of ions with their atmosphere due to a response described by $\boldsymbol{\kappa}$. We may merely replace $\boldsymbol{\kappa}$ by $\boldsymbol{\kappa}^{\prime}$ and $D_{s}$ by $D_{s}^{\prime}$ under the approximations stated.
} 
was called $\Delta F^{*}{ }_{\text {sol. }}$. In (89) to (92) we have omitted the prime superscripts for brevity.

In the case of electrode systems, there is only one ion, but there is also the image charge of opposite sign in the electrode. ${ }^{28}$

Instead of (A24) to (A26) one finds,

(electrode) $F^{\mathrm{op}}{ }_{m(r-p)}=-\frac{(m \Delta e)^{2}}{2 a}\left(1-\frac{1}{D_{\mathrm{op}}^{\prime}}\right)-\frac{(m \Delta e)^{2}}{2 D_{\mathrm{op}}^{\prime} R}$

and that $F_{m(r-p)}$ is the sum of (A28) and of one-half (A26),

$$
-\frac{(m \Delta e)^{2}}{2 a}\left(1-\frac{1}{D_{s}^{\prime}}\right)-\frac{(m \Delta e)^{2}}{2 D_{s}^{\prime} R} .
$$

In this way (90) and (92) of the text were obtained.

\section{APPENDIX VIII. CORRELATIONS OF OVER-ALL RATE CONSTANTS}

Equations (31), (33), (81), and (82) describe the rate constant for any reactants with intact, specified inner coordination shells. $\Delta F^{\circ \prime}$ there refers to the change for those species. Consider now the rate constants expressed in terms of the stoichiometric concentration of each redox reagent. The region of (81) linear in $\Delta F^{\circ}$ is the most important one in terms of the correlations made in Part $\mathrm{V}$, and we restrict our attention here to such cases for each elementary redox step (A29) below. We consider only the case where the dissociation or formation of any important complex does not contribute appreciably to the reaction coordinate near the intersection surface: We make use of (81) and note that its derivation was based on intact coordination shells in a system near the intersection surface; the properties of the "reactants" or "products" appearing in Eq. (81) refer to those with such shells, even though they might be unstable.

We consider the homogeneous case first. Let $\mathbf{m}$ denote the totality of any ligands $X_{1}, X_{2}, \cdots$ in a reacting member of the $A$ redox system having $m_{i}$ ligands of Type $X_{i}$,

$$
\mathbf{m}=\left(m_{1}, m_{2}, \cdots, m_{i}, \cdots\right) .
$$

Let $n$ play the same role for the $B$ system

$$
\mathbf{n}=\left(n_{1}, n_{2}, \cdots, n_{i}, \cdots\right) \text {. }
$$

Let the reactants and products be denoted by $r$ and $p$ superscripts, respectively. A typical contribution to the over-all redox reaction is (A29). Let it have a bimolecular rate constant $k_{m n}$ for the forward step

$$
A_{m}{ }^{r}+B_{n}{ }^{r} \stackrel{k m n^{r}}{\longrightarrow} A_{m}^{p}+B_{n}{ }^{p} \text {. }
$$

The over-all second-order rate constant $k_{a b}$ then involves a weighted sum over the rates of all bimolecular $m n$ contributions, per unit stoichiometric concentrations of $A^{r}$ and of $B^{r}$ :

$$
k_{a b}=\sum_{m, n} k_{m n}{ }^{r}\left(A_{m}{ }^{r}\right)\left(B_{n}^{r}\right) / \sum_{m}\left(A_{m}{ }^{r}\right) \sum_{n}\left(B_{n}^{r}\right),
$$

where ( ) denotes concentration. If $\pi_{m}{ }^{r}$ and $\pi_{n}{ }^{r}$ denote the probabilities that an $A^{r}$ species exists as $A_{m}{ }^{r}$ and that a $B^{r}$ one exists as $B_{n}{ }^{r}$, respectively, i.e., if

$$
\pi_{m}^{r}=\left(A_{m}^{r}\right) / \sum_{m}\left(A_{m}^{r}\right), \quad \pi_{n}^{r}=\left(B_{n}^{r}\right) / \sum_{n}\left(B_{n}^{r}\right),
$$

then (A23) becomes

$$
k_{a b}=\sum_{m, n} k_{m n}{ }^{r} \pi_{m}{ }^{r} \pi_{n}{ }^{r} .
$$

Let $F_{m}{ }^{r}+F_{n}{ }^{r}$ denote the free energy of the system containing a labeled $A_{m}{ }^{r}$ and a labeled $B_{n}{ }^{r}$ molecule far from each other, fixed in the medium, under the prevailing conditions. Let the corresponding property be $F_{m}{ }^{p}+F_{n}{ }^{p}$ when the two labeled molecules are $A_{m}{ }^{p}$ and $B_{n}{ }^{p}$. We subdivide $F_{m}{ }^{r}+F_{n}{ }^{r}$ such that $F_{m}{ }^{r}$ depends on the properties of $A_{m}{ }^{r}$ and its environment alone. It is therefore independent of the nature of $B_{n}{ }^{r}$. We note that the $\pi$ 's can be expressed in terms of these $F$ 's, if we assume, as we do, that the complexes $A_{m}{ }^{r}$ and $B_{n}{ }^{r}$ have an equilibrium population,

$$
\pi_{m}^{r}=\frac{\exp \left(-F_{m}{ }^{r} / k T\right)}{\sum_{m} \exp \left(-F_{m}^{r} / k T\right)}, \text { etc. }
$$

In virtue of their definition these $F$ 's depend on the concentration of $X_{i}$ 's. The free energy of any reaction (A32) in the prevailing medium is in fact $F_{m}{ }^{r}-F_{m}{ }^{r}$ :

$$
A_{m}{ }^{r}+\sum_{i}\left(m_{i}^{\prime}-m_{i}\right) X_{\imath} \rightarrow A_{m}{ }^{r} \text {. }
$$

Each $k_{m n}$ is given by a pair of equations of the type (31), (81), where for $\lambda$ we write $\lambda_{m n}$ and recall the additivity of $\lambda$

$$
\lambda_{m n}=\lambda_{m}+\lambda_{n} \text {. }
$$

On using (A32) the $\Delta F^{\circ \prime}$ for Step (A29) is seen to be

$$
\Delta F_{m n}{ }^{\circ}=F_{m}^{p}+F_{n}{ }^{p}-F_{m}{ }^{r}-F_{n}{ }^{r} .
$$

On neglecting $\Delta F_{m n}{ }^{0 / 2} / 4 \lambda_{m n}$ in (81) as discussed earlier one obtains (A35), using (A30) to (A34):

$$
\begin{array}{r}
k_{a b}=Z K_{a b^{\frac{1}{2}}} \sum_{m, n} \exp \left\{-\left[w_{m n}{ }^{r}+w_{m n}{ }^{p}+\frac{1}{2}\left(\lambda_{m}+\lambda_{n}\right)\right] / 2 k T\right\} \\
\left.\times\left(\pi_{m}^{p} \pi_{m}{ }^{r} \pi_{n}^{p} \pi_{n}^{r}\right)^{\frac{1}{2}}, \quad \text { ( } \mathrm{A} 35\right)
\end{array}
$$

where $K_{a b}$ is given by (A36) and is, in fact, easily demonstrated to be the formal equilibrium constant of the reaction in the given medium, expressed in terms of the stoichiometric concentrations

$$
K_{a b}=\frac{\sum_{m} \exp \left(-F_{m}^{p} / k T\right) \sum_{n} \exp \left(-F_{n}^{p} / k T\right)}{\sum_{m} \exp \left(-F_{m}^{r} / k T\right) \sum_{n} \exp \left(-F_{n}^{r} / k T\right)} .
$$


This equilibrium constant is, by definition,

$$
\sum_{m}\left(A_{m}^{p}\right) \sum_{n}\left(B_{n}^{p}\right) / \sum_{m}\left(A_{m}{ }^{r}\right) \sum_{n}\left(B_{n}{ }^{r}\right) .
$$

From (A35) one can at once derive an expression for the isotopic exchange rate constant. On considering the $A$ redox system a typical contribution to the exchange will be (A37) when $m$ and $m^{\prime}$ describe any two complexes. The over-all rate constant, $k_{a a}$, is then obtained by multiplying $k_{m m}{ }^{r}$ by $\pi_{m}{ }^{r} \pi_{m^{\prime}}{ }^{p}$ and summing over all $m$ and $m^{\prime}$. The result is given by (A38), and is then counterpart of (A35):

$$
\begin{gathered}
A_{m}{ }^{r}+A_{m^{\prime}} \stackrel{{ }^{p m m^{\prime}} \stackrel{{ }^{r}}{\longrightarrow} A_{m}{ }^{p}+A_{m^{\prime}}{ }^{r},}{k_{a a}=\sum_{m, m^{\prime}} k_{m m^{\prime}}{ }^{r} \pi_{m}{ }^{r} \pi_{m^{\prime}}{ }^{p} .}
\end{gathered}
$$

$k_{a a}$ is obtained from (A35) by noting that $K_{a a}$ is unity

$$
\begin{array}{r}
k_{a a}=Z \sum_{m, m^{\prime}} \exp \left\{-\left[w_{m m^{\prime}}{ }^{r}+w_{m m^{\prime}}{ }^{p}+\frac{1}{2}\left(\lambda_{m}+\lambda_{m^{\prime}}\right)\right] / k T\right\} \\
\times\left(\pi_{m}{ }^{p} \pi_{m}{ }^{r} \pi_{m^{\prime}}{ }^{p} \pi_{m^{\prime}}{ }^{r}\right) .
\end{array}
$$

When the work terms can be neglected one finds

$$
\begin{gathered}
k_{a b}=Z K_{a b^{\frac{1}{2}}} \sum_{m} \exp \left(-\lambda_{m} / 4 k T\right)\left(\pi_{m}^{p} \pi_{m}^{r}\right)^{\frac{1}{2}} \\
\sum_{n} \exp \left(-\lambda_{n} / 4 k T\right)\left(\pi_{n}^{p} \pi_{n}^{r}\right)^{\frac{1}{2}}, \\
k_{a a}=Z\left[\sum_{m} \exp \left(-\lambda_{m} / 4 k T\right)\left(\pi_{m}^{p} \pi_{m}^{r}\right)^{\frac{1}{j}}\right]^{2} .
\end{gathered}
$$

From (A40) and (A41) one then obtains

$$
k_{a b}=\left(k_{a a} k_{b b} K_{a b}\right)^{\frac{3}{3}} \text {. }
$$

On considering next the electrochemical case, let $M$ denote the electrode, $M^{r}$ describing its state before electron transfer and $M^{p}$ after. As in the text we assume that the acquisition or loss of an electron by the electrode has essentially no effect on the force constants or equilibrium bond distances in any adsorbed layer of ions or molecules. (To be sure, one or more electrons on the electrode may be fairly localized when the reacting species is near it, and this number changes when the species gains or loses electrons.) We regard different compositions of the adsorbed layer as corresponding to different domains of the coordinates in many-dimensional space.

The free energy of a system having a labeled $A_{m}{ }^{r}$ molecule far from the electrode and fixed in position is written as $F_{m}{ }^{r}+F_{M^{r}}$, the corresponding term when the molecule is $A_{m}{ }^{p}$ (and the electrode has lost $n$ electrons thereby) is $F_{m}^{p}+F_{M}{ }^{p}$. The free energy of Reaction (73) for the case where the reactant is $A_{m}{ }^{r}$ is then given by (A43), since the translational contribution for $A_{m}$ cancels in computing $F_{m}{ }^{p}-F_{m}{ }^{r}$. The change depends linearly on $E$, as in (74). $F_{M}{ }^{p}$ and $F_{M}{ }^{r}$ are independent of the properties of $A$. They depend only on those of the electrode and the electrical double-layer region

$$
F_{m}{ }^{p}+F_{M^{p}}-F_{m}{ }^{r}-F_{M}{ }^{r}=\Delta_{m}+n e E,
$$

where $\Delta_{m}$ is independent of $E$.

When electrochemical equilibrium exists ( $E$ equals $E_{\theta}$ then), it does so for each $m$. Adding to the free energy difference (A43) the mixing term, $k T \ln \left(A_{m}{ }^{p}\right) /\left(A_{m}{ }^{r}\right)$, the result must equal zero at equilibrium. We thereby obtain from (A43) the value of each $\Delta_{m}$,

$$
\Delta_{m}=-n e E_{e}-k T \ln \left(A_{m}^{p}\right) /\left(A_{m}{ }^{r}\right) .
$$

Equation (A45) is finally obtained for the free-energy difference

$$
\begin{aligned}
F_{m}{ }^{p}+F_{M^{p}}{ }^{p}-F_{m}{ }^{r}-F_{M}{ }^{r}= & n e\left(E-E_{e}\right) \\
& -k T \ln \left(A_{m}^{p}\right) /\left(A_{m}{ }^{r}\right) .
\end{aligned}
$$

Utilizing the fact that $E_{e}$ is related to $E_{0}{ }^{\prime}$ according to (75), where (Ox) now equals $\sum_{m}\left(A_{m}^{p}\right)$ and (Red) equals $\sum_{m}\left(A_{m}{ }^{r}\right)$, (A45) can be rewritten as

$F_{m}^{p}+F_{M^{p}}-F_{m}{ }^{r}-F_{M^{r}}=n e\left(E-E_{o}{ }^{\prime}\right)-k T \ln \pi_{m}{ }^{p} / \pi_{m}{ }^{r}$.

From (A31) and (A46) one obtains:

$$
\begin{aligned}
\exp \left[-n e\left(E-E_{o}{ }^{\prime}\right) / k T\right) & =\exp \left[-\left(F_{M^{p}}-F_{M^{r}}\right) / k T\right] \\
& \times \frac{\sum_{m} \exp \left(-F_{m}{ }^{p} / k T\right)}{\sum_{m} \exp \left(-F_{m}{ }^{r} / k T\right)} \cdot
\end{aligned}
$$

For the over-all electrochemical rate constant of the forward reaction in (73), $k_{\mathrm{el}}$, we have

$$
k_{\mathrm{el}}=\sum_{m} k_{m}{ }^{r} \pi_{m}{ }^{r},
$$

where $k_{m}{ }^{r}$ is the rate constant for $\left(A_{m}^{r}\right)$ going to $\left(A_{m}^{p}\right)$ at the given $E$. For each $m$, the $k_{m}{ }^{r}$ is given by an equation analogous to (82), with $n e\left(E-E_{o}{ }^{\prime}\right)$ replaced by $n e\left(E-E_{o}{ }^{\prime}\right)-k T \ln \pi_{m}{ }^{p} / \pi_{m}{ }^{r}$ [compare Eqs. (77) and (A46)]. One then obtains

$$
\begin{aligned}
k_{\mathrm{el}}= & Z_{\mathrm{el}} \exp \left[-n e\left(E-E_{o}^{\prime}\right) / 2 k T\right] \\
& \times \sum_{m} \exp \left[-\left(w_{m}^{r}+w_{m}^{p}+\frac{1}{2} \lambda_{m}\right) / 2 k T\right]\left(\pi_{m}^{p} \pi_{m}^{r}\right)^{1} .
\end{aligned}
$$

The work terms naturally depend on $E$. When they can be neglected one has

$$
\begin{aligned}
k_{\mathrm{el}}=Z_{\mathrm{el}} \exp [- & \left.n e\left(E-E_{o}{ }^{\prime}\right) / 2 k T\right] \\
& \times \sum_{m} \exp \left(-\lambda_{m} / 4 k T\right)\left(\pi_{m}{ }^{p} \pi_{m}{ }^{r}\right)^{\frac{3}{3}} .
\end{aligned}
$$

In the light of Eqs. (A40) to (A42), (A49), and (A50), we see that the correlations (a) to (f) in Sec. 18 
still hold, even when applied to over-all rate constants but, as one sees from (A42), (a) is now restricted to the region of chemical transfer coefficient equal to $\frac{1}{2}$ [i.e., to $f \sim 1$ in (96) ].

\section{APPENDIX IX. ALTERNATIVE DERIVATION OF (13)}

As we have seen in the text, the configurational distribution of the $V^{\prime}{ }_{i}$ and $V^{\prime}{ }_{0}$ coordinates in the activated complex is not one which is appropriate to Surface $R$ nor one appropriate to Surface $P$. That is, it is appropriate to neither electronic structure (the initial or final one) of a reacting species. Cognizance of this nonequilibrium distribution of solvent molecules was taken in Part I, using a dielectric continuum treatment of systems possessing nonequilibrium dielectric polarization. An expression for the free energy of a system with arbitrary polarization was minimized, subject to an energy equation of constraint, the dielectric continuum counterpart of (20). In this Appendix we show that this method, formulated now in terms of statistical mechanics yields the same result as the method used in Appendix II.

The configurational contribution to free energy of a nonequilibrium system described by a potential energy $U^{r}$ and a distribution function $f^{*}$, where $f^{*}$ is to be determined, is given by (A51) to an additive constant

$$
F^{\mathrm{non}}=\int f^{*} U^{r} d V^{\prime}+k T \int f^{*} \ln f^{*} d V^{\prime}
$$

Minimizing (A51) subject to the energy equation of constraint (A52) and to (A53),

$$
\begin{gathered}
\int\left(U^{r}-U^{p}\right) f^{*} d V^{*}=0, \\
\int f^{*} d V^{*}=1,
\end{gathered}
$$

we obtain (A54), where $\alpha$ and $m$ are Lagrangian multipliers:

$$
\int\left(U^{r}+m\left(U^{r}-U^{p}\right)+k T \ln f^{*}+\alpha\right) \delta f^{*} d V^{\prime}=0 .
$$

Setting the coefficient of $\delta f^{*}$ equal to zero, and evaluating $\alpha$ from (A52) we find

$$
f^{*}=\exp \left(-\frac{U^{*}}{k T}\right) / \int \exp \left(-\frac{U^{*}}{k T}\right) d V^{\prime},
$$

where $U^{*}$ equals $U^{r}+m\left(U^{r}-U^{p}\right)$. This equation was also obtained by the method in Appendix II. Once again, $m$ is determined by the energy condition (A52).

\title{
Current Oscillations in Solid Polystyrene and Polystyrene Solutions*
}

\author{
A. Weinreb, N. Ohana, and A. A. Braner \\ Department of Physics, The Hebrew University of Jerusalem, Israel
}

(Received 19 March 1965)

\begin{abstract}
Application of a dc voltage across a plate of polystyrene gives rise to oscillatory currents which reach considerably high negative values. The dependence of current intensity (maximum, minimum, and plateau values) on various parameters (voltage, dimensions of samples and electrodes, nature of dissolved solute, etc., as well as repetitive use) is treated. The pattern of oscillation is found to depend on all these parameters, too. The length of the oscillation period decreases very quickly with increasing voltage. It depends also very strongly on the nature and pressure of the surrounding gas.
\end{abstract}

\section{INTRODUCTION}

$\mathbf{I}^{\mathrm{N}}$ $\mathrm{N}$ trying to measure the extremely low dark conductivity of polystyrene we found a prohibitively strong influence of air on the measured intensities of the currents. The variation in current intensity which usually follows any mechanical handling of a plastic was also found to be strongly influenced by the presence of air. In order to avoid the effect of air the "chamber"

* Performed under the auspices of the U. S. Atomic Energy Commission, Contract NYO-2949-6. which houses the investigated specimen was evacuated. Upon evacuation the following effect was observed: The current oscillates with a definite pattern reaching high negative values although a dc voltage is applied. ${ }^{1}$ The period of oscillation as well as its pattern depends strongly on the voltage. It depends also strongly on the nature and pressure of the surrounding gas. These oscillations present a serious obstacle in measuring

1 A. Weinreb, N. Ohana, and A. A. Braner, Phys. Letters 10, 278 (1964). 